\title{
The Effects of Working Memory, Perceptual Speed, and Inhibition in Aggregated Search
}

\author{
JAIME ARGUELLO and BOGEUM CHOI, School of Information and Library Science, \\ University of North Carolina at Chapel Hill
}

\begin{abstract}
Prior work has studied how different characteristics of individual users (e.g., personality traits and cognitive abilities) can impact search behaviors and outcomes. We report on a laboratory study $(N=32)$ that investigated the effects of three different cognitive abilities (perceptual speed, working memory, and inhibition) in the context of aggregated search. Aggregated search systems combine results from multiple heterogeneous sources (or verticals) in a unified presentation. Participants in our study interacted with two different aggregated search interfaces (a within-subjects design) that differed based on the extent to which the layout distinguished between results originating from different verticals. The interleaved interface merged results from different verticals in a fairly unconstrained fashion. Conversely, the blocked interface displayed results from the same vertical as a group, displayed each group of vertical results in the same region on the SERP for every query, and used a border around each group of vertical results to help distinguish among results from different sources. We investigated three research questions (RQ1-RQ3). Specifically, we investigated the effects of the interface condition and each cognitive ability on three types of outcomes: (RQ1) participants' levels of workload, (RQ2) participants' levels of user engagement, and (RQ3) participants' search behaviors. Our results found different main and interaction effects. Perceptual speed and inhibition did not significantly affect participants' workload and user engagement but significantly affected their search behaviors. Specifically, with the interleaved interface, participants with lower perceptual speed had more difficulty finding relevant results on the SERP, and participants with lower inhibitory attention control searched at a slower pace. Working memory did not have a strong effect on participants' behaviors but had several significant effects on the levels of workload and user engagement reported by participants. Specifically, participants with lower working memory reported higher levels of workload and lower levels of user engagement. We discuss implications of our results for designing aggregated search interfaces that are well suited for users with different cognitive abilities.
\end{abstract}

CCS Concepts: • Information systems $\rightarrow$ Users and interactive retrieval; Search interfaces;

Additional Key Words and Phrases: Cognitive abilities, perceptual speed, working memory, inhibition, aggregated search, workload, user engagement, search behaviors

ACM Reference format:

Jaime Arguello and Bogeum Choi. 2019. The Effects of Working Memory, Perceptual Speed, and Inhibition in Aggregated Search. ACM Trans. Inf. Syst. 37, 3, Article 36 (May 2019), 34 pages.

https://doi.org/10.1145/3322128

This work was supported by NSF grant IIS-1451668. Any opinions, findings, conclusions, and recommendations expressed in this article are those of the authors and do not necessarily reflect the views of the sponsor.

Authors' addresses: J. Arguello and B. Choi, 216 Lenoir Dr, Chapel Hill, NC 27599.

Permission to make digital or hard copies of all or part of this work for personal or classroom use is granted without fee provided that copies are not made or distributed for profit or commercial advantage and that copies bear this notice and the full citation on the first page. Copyrights for components of this work owned by others than ACM must be honored. Abstracting with credit is permitted. To copy otherwise, or republish, to post on servers or to redistribute to lists, requires prior specific permission and/or a fee. Request permissions from permissions@acm.org.

(C) 2019 Association for Computing Machinery.

1046-8188/2019/05-ART36 \$15.00

https://doi.org/10.1145/3322128

ACM Transactions on Information Systems, Vol. 37, No. 3, Article 36. Publication date: May 2019. 


\section{INTRODUCTION AND MOTIVATION}

Information retrieval systems are intended to support users with a wide range of characteristics. Therefore, an important research question is as follows: How do characteristics of individual users influence their search behaviors and outcomes? Prior studies in interactive information retrieval (IIR) have explored this question and have focused on personality traits such as need for cognition (NFC) and cognitive abilities such as perceptual speed (PS) [1-3, 14, 54] and working memory (WM) [24, 27, 29, 40, 43]. Studies have found that such characteristics of individual users can have important effects. For example, with respect to need for cognition (i.e., a user's predisposition to enjoy cognitively demanding activities), Wu et al. [56] found that high-NFC participants were less likely to examine search results at lower ranks and more likely to formulate new queries. With respect to perceptual speed, Brennan et al. [14] found that high-PS participants experienced lower levels of workload while completing search tasks. Finally, with respect to working memory, Gwizdka [29] found that high-WM participants exerted more search effort (i.e., were less likely to satisfice) in demanding situations.

We report on a laboratory study $(N=32)$ that investigated the effects of three cognitive abilities: (1) working memory, (2) perceptual speed, and (3) inhibition. Broadly speaking, working memory refers to someone's ability to hold information in an active state [19]; perceptual speed refers to someone's ability to quickly scan a visual display for information [22]; and inhibitory attention control, or simply inhibition, refers to someone's ability to ignore (or inhibit attention to) stimuli that are not relevant to the task at hand [19]. Our study focused on these three cognitive abilities in the context of aggregated search.

Aggregated search is concerned with developing systems that integrate results from multiple heterogeneous sources in a unified presentation [4]. Web search portals such as Google and Bing are examples of aggregated search systems. In addition to returning web pages, web search portals integrate results from specialized back-end systems (or verticals) that focus on specific types of media (e.g., images, videos) and search intents (e.g., news, shopping). Web search portals are perhaps the most widely used aggregated search systems. However, aggregated search systems are not limited to the web search domain. Other examples include scientific search systems, ${ }^{1}$ library search systems, ${ }^{2}$ and social media search systems. ${ }^{3}$ Furthermore, aggregated search techniques have been applied to desktop search [36], which requires searching different types of files.

Developing an aggregated search system requires deciding how to display results originating from different back-end systems. Namely, to what extent should the interface help distinguish between different types of results? For example, to what extent should different types of results use different surrogate representations? Should results of the same type be displayed as a group or be interleaved with other types of results? To what extent should the interface use visual cues (e.g., colored backgrounds and/or borders) to further distinguish between different types of results?

To illustrate, Figure 1 shows three aggregated search systems that use different presentation layouts. At the time of this writing, PubMed (Figure 1(a)) shows articles prominently in the center and extracted images off to the side and always in the same position. The UNC Libraries search system (Figure 1(b)) shows digital materials on the left and physical materials on the right. Within each block, different types of materials (e.g., online articles, e-books, books, microforms) are intermixed (i.e., not clearly distinguished from each other). Finally, Facebook search (Figure 1(c)) shows different types of results (e.g., pages, groups, articles, posts) stacked vertically. Some results

\footnotetext{
${ }^{1}$ PubMed provides aggregated search across articles and extracted images.

${ }^{2}$ The UNC Libraries search system provides aggregated search across different types of digital materials (e.g., articles, e-books) and physical materials (e.g., books, CDs, microforms).

${ }^{3}$ Facebook provides aggregated search across posts, people, groups, photos, videos, apps, and external links.
} 


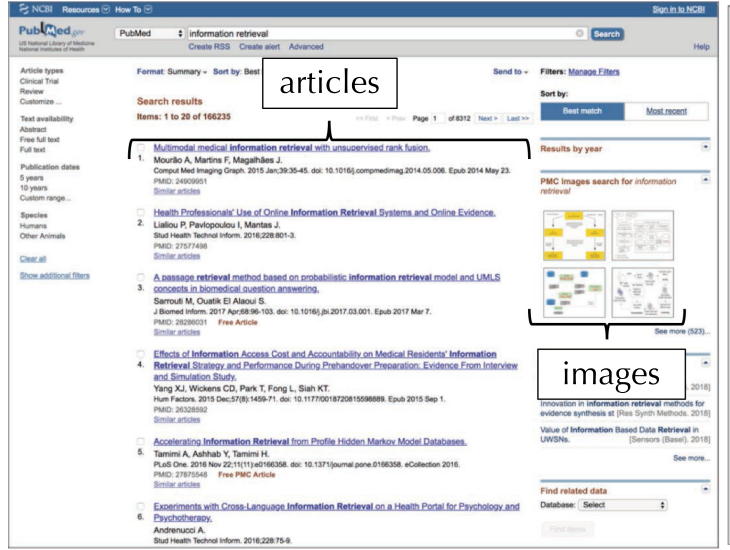

(a) PubMed (truncated)

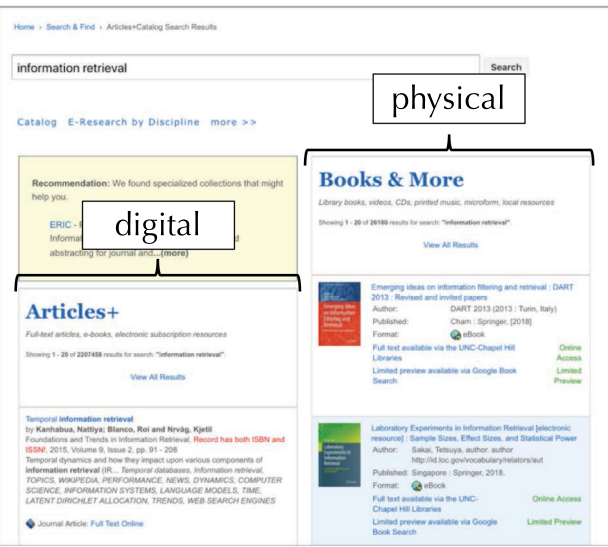

(b) UNC Libraries (truncated)

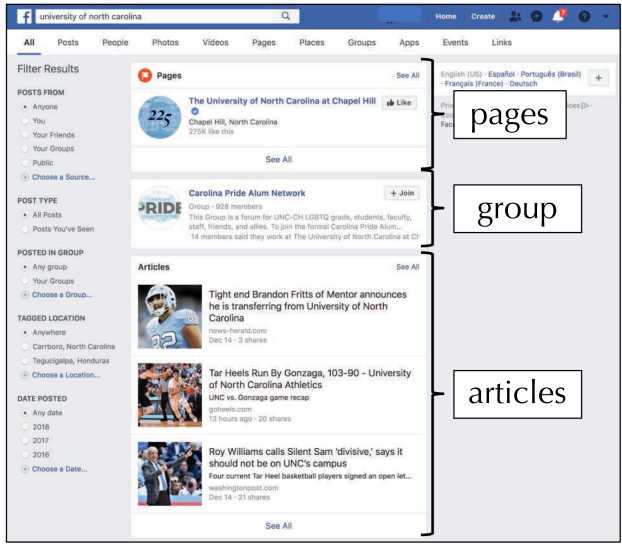

(c) Facebook (truncated)

Fig. 1. Aggregated search interfaces differ in the extent to which the presentation layout distinguishes between different types of results.

are grouped (e.g., articles) and others are individually interleaved (e.g., group pages). These examples illustrate how aggregated search systems differ in the extent to which the visual layout distinguishes between different result types. In this study, our goal was to gain insights into how these design considerations may impact users with different cognitive abilities.

We report on a laboratory study in which participants completed four search tasks using two aggregated search interfaces. Given the same query, both interfaces displayed the same results but differed in the extent to which the visual layout clearly marked results from different sources. The interleaved interface combined results from different verticals (web, images, news, shopping, videos) in a fairly unconstrained fashion. In contrast, the blocked interface presented results from the same vertical as a group, always presented groups in the same regions on the SERP, and used visual cues (i.e., borders with a shadow effect) to help distinguish between results from different verticals. After completing all four tasks, participants completed three psychometric tests to measure their working memory ability, perceptual speed, and inhibitory attention control.

In this study, we investigated three research questions (RQ1-RQ3). Specifically, we investigated the effects of the aggregated search interface condition (interleaved vs. blocked) and each 
cognitive ability (working memory, perceptual speed, and inhibition) on (RQ1) participants' reported levels of workload, (RQ2) user engagement, and (RQ3) search behaviors. To address RQ1, we used items from the raw NASA-TLX questionnaire [30] to measure different aspects of workload (e.g., mental, physical, and temporal demand). To address RQ2, we used O'Brien's User Engagement Scale [46] to measure different factors of user engagement (e.g., focused attention, perceived usability, and aesthetic appeal). ${ }^{4}$ Finally, to address RQ3, we logged participants' interactions with both aggregated search interfaces and computed different measures associated with their level of search activity, trial-and-error, and pace of interaction.

While there are many cognitive abilities, we considered three that are likely to influence behaviors and outcomes within aggregated search. Prior IIR studies have considered the effects of working memory and perceptual speed [27, 29, 54]. In terms of working memory (WM), studies have found that low-WM participants are more likely to satisfice in demanding situations [27, 29]. In this respect, low-WM users may be more likely to satisfice while interacting with a more visually complex aggregated search layout. In terms of perceptual speed (PS), Turpin et al. [54] studied high- and low-PS participants in the context of aggregated search. Participants interacted with two systems: an aggregated and a non-aggregated system. High-PS participants took about the same amount of time to complete tasks with both systems, but low-PS participants took longer with the aggregated system. In this respect, low-PS users may have more difficulty scanning for relevant results with a more visually complex layout. Finally, to our knowledge, IIR studies have not directly considered the effects of inhibitory attention control. However, we expected that a searcher's ability to ignore (or inhibit attention to) non-relevant results (e.g., results from one or more non-relevant verticals) might play an important role in aggregated search. Prior work has not investigated how different aggregated search layouts may influence users with different abilities.

Furthermore, it is worth pointing out that deficits in working memory, perceptual speed, and inhibition are not uncommon. For example, deficits in working memory have been found in people with attention-deficit/hyperactivity disorder (ADHD) [33], which affects an estimated $10 \%$ of U.S. children ages 3-17 [47]. Similarly, deficits in inhibitory attention control have been found in people with dyslexia [32], which accounts for an estimated $15-20 \%$ of the U.S. population [10]. Additionally, studies have found that aging is associated with deficits in working memory [12] and attention control [31]. In light of these trends, it seems worthwhile to further understand the impact of these cognitive abilities on searchers.

Understanding how cognitive abilities influence behaviors and outcomes has several important implications. First, it can help us design systems that are more accessible for users with a wide range of abilities. Second, it can inform the design of personalized presentations, interactions, and assistance tools to support users based on their unique abilities. Third, it can help us more accurately interpret search behaviors from individual users (i.e., implicit feedback signals) to predict outcomes (e.g., user satisfaction). Finally, it can provide insights into how to infer the abilities of individual users from behavioral data and help us understand which abilities may be easier (or more difficult) to infer.

\section{RELATED WORK}

Our research builds on five areas of prior work. We extend prior research on the effects of working memory, perceptual speed, and inhibition on search behaviors and outcomes. Additionally, we build on prior studies of search behavior within aggregated search. Finally, we build on early work on Gestalt principles that help explain how users perceive relations between items in an information display such as an aggregated search results page (SERP).

\footnotetext{
${ }^{4}$ Workload and engagement were measured after the participant completed each search task.
} 


\subsection{Working Memory}

Working memory (WM) is defined as someone's ability to hold information in short-term memory when it is no longer perceptually present [19]. Working memory is considered a core executive function and is critical for making sense of anything that unfolds over time [19]. In this respect, working memory impacts higher-level tasks such as reading, following instructions, incorporating new information into action plans, identifying relations, and deriving general principles from particular observations [19].

Several studies have considered the impact of working memory on search behavior. Gwizdka [27] investigated the interplay between two cognitive abilities (verbal closure and memory span), search task complexity, and two different interfaces. Both interfaces returned a ranked list of search results, but one interface (the overview interface) also presented a summary of the results using a word cloud with clickable terms to filter the search results. In less-demanding situations (i.e., during simple tasks using the overview system), high-WM participants completed tasks faster than low-WM participants. Conversely, in more-demanding situations (i.e., during complex tasks using the baseline system), high-WM participants exerted more effort than low-WM participants. In other words, low-WM participants were more likely to satisfice in demanding situations. In a later study, Gwizdka [29] used eye-tracking to compare the search activities of high- and low-WM participants while completing complex search tasks. High- and low-WM participants had similar task completion times, but high-WM participants performed more actions during the search session. Furthermore, based on eye-tracking data, high-WM participants spent more time reading pages than low-WM participants, particularly toward the end of the search session. Consistent with previous findings [27], these results suggest that low-WM participants may be more likely to satisfice in demanding situations.

Prior studies have also compared the behaviors of dyslexic and non-dyslexic searchers [24, 40, 43]. While dyslexia is a heterogeneous condition, evidence suggests that people with dyslexia may exhibit lower levels of phonological working memory (i.e., the ability to hold words in short-term memory). MacFarlane et al. [40] compared the search behaviors of dyslexic and non-dyslexic students and found that non-dyslexic students examined more documents and judged a greater number (and percentage) of documents as being non-relevant. In other words, non-dyslexic students judged more documents and were more "selective" in their relevance judgements.

Morris et al. [43] studied the challenges faced by dyslexic searchers through a series of in-depth interviews, an online questionnaire, and a laboratory study. Results found that dyslexic searchers prefer search results with more multimedia items (e.g., images), less clutter (e.g., fewer ads), less "density" of text (e.g., shorter sentences), and more structural elements (e.g., titles, headings, lists, tables). Furthermore, the authors conducted a study in which dyslexic and non-dyslexic participants were asked to judge a set of webpages along different dimensions of readability and understandability. Responses from dyslexic participants were less positive than non-dyslexic participants.

Fourney et al. [24] analyzed the differences between readability and relevance judgments produced by dyslexic and non-dyslexic study participants. Results found three important trends. First, participants' readability and relevance judgments were highly correlated, suggesting that readability is an important relevance criterion. Second, regression models using only 14 webpage attributes (e.g., avg. line length, avg. image size, avg. font size, and number of structural elements) were able to explain about $12-16 \%$ of the variance in the readability and relevance judgments from both participant groups. Finally, the relevance judgments from dyslexic participants had a greater central tendency (i.e., were less bimodal), suggesting that dyslexic participants had more difficulty distinguishing between relevant and non-relevant pages. In other words, consistent with MacFarlane et al. [40], dyslexic participants were less "selective" in their relevance judgments. 


\subsection{Perceptual Speed}

Perceptual speed (PS) is defined as someone's speed in comparing figures or symbols, scanning to find figures or symbols, and performing other simple tasks involving visual perception [22]. It has also been defined as someone's ability to compare visual patterns or identify a visual pattern among distracting patterns [49]. Perceptual speed is considered an automatic mental process [22] and has been found to impact higher-level executive functions such as working memory [23].

Prior studies in the area of IIR have found that perceptual speed (PS) can influence different search behaviors and outcomes. In an early study by Allen [2], high-PS participants were more effective than low-PS participants at finding relevant material (based on precision and recall). Allen conjectured that high-PS participants were more effective at identifying useful sources and vocabulary to use in subsequent searches. In a later study, Allen [3] investigated the mechanisms through which high-PS users outperform low-PS users. Allen introduced a system manipulation in which the subject headings of the bibliographic search results returned to users were more visually salient as compared to a baseline system. High-PS participants were better able to use this system feature to improve their search performance, while low-PS participants showed no difference in performance across systems. Kim and Allen [37] conducted one of the first studies to examine the influence of three cognitive abilities in the context of web search: perceptual speed, spatial scanning ability (SSA), and logical reasoning ability (LRA). While perceptual speed did not have an effect on participants' behaviors, SSA (a closely related cognitive ability) had a significant interaction effect with the task topic. Specifically, while completing search tasks related to their occupation (instead of less familiar tasks), high-SSA participants viewed and bookmarked more pages than low-SSA participants.

Al-Maskari and Sanderson [1] investigated the influence of search experience and perceptual speed on search performance. High- and low-PS participants performed equally well in terms of the number of relevant documents found, but high-PS participants took significantly less time to find the first relevant document. Brennan et al. [14] investigated the effects of perceptual speed and task complexity on participants' levels of workload and search behaviors. High-PS participants reported significantly lower levels of workload and interacted with greater intensity than low-PS participants (e.g., had shorter completion times, more SERP clicks, and more pages visited).

Closely related to our work, Turpin et al. [54] investigated the influence of perceptual speed in the context of aggregated search. Participants interacted with two different interfaces: (1) a blended system that combined results from different verticals into the main SERP and (2) a non-blended system that presented results from different verticals on different tabs (not aggregated). Irrespective of the interface, high-PS participants reported greater levels of satisfaction with the system and their own performance than low-PS participants. Furthermore, perceptual speed and interface had a significant interaction effect on the task completion time-high-PS participants took roughly the same amount of time with both interfaces, but low-PS participants took significantly longer with the blended interface (the more visually "busy"). Whereas Turpin et al. [54] compared between an aggregated and non-aggregated system, in this article we compare between two aggregated layouts.

\subsection{Inhibition}

Broadly speaking, inhibition is defined as someone's ability to control his or her attention, behavior, thoughts, and/or emotions by overriding an internal predisposition or external "lure" and instead remaining focused on what is appropriate or needed [19]. Specifically, in this article, we focus on inhibitory attention control, which refers to someone's ability to suppress their attention to stimuli that are not relevant to the task at hand. In this respect, people with high inhibitory 
attention control are more effective at strategically ignoring (or inhibiting attention to) specific stimuli based on their intentions and goals [19].

To our knowledge, prior IIR studies have not directly investigated the influence of inhibition on search behaviors and outcomes. One study by Gwizdka [28] used attention control on a secondary task as a means to measure workload during a primary task. The goal of this study was to compare the levels of workload experienced during different search-related activities (e.g., while formulating queries, evaluating search results, reading content pages, etc.). While conducting searches (the primary task), participants were given short Stroop tests at random intervals (the secondary task). ${ }^{5}$ Participants' performance on these secondary Stroop tests varied during different search activities, suggesting that different search activities require different amounts of mental effort. This study was not aimed at investigating the influence of participants' inhibitory attention control on their search behaviors. However, results from this study suggest that mental workload (e.g., during multitasking) is one contextual factor that may impact someone's performance on tasks requiring attention control.

\subsection{Aggregated Search}

Aggregated search is concerned with designing systems that combine results from multiple heterogeneous sources in a unified presentation [4]. Commercial search portals such as Google and Bing are examples of aggregated search systems. In addition to returning web results, commercial search portals integrate results from other back-end systems (or verticals) into the main SERP. Common verticals include systems that search for images, news articles, products for sale, and videos. A large body of prior work has focused on developing algorithms for different predictive tasks associated with aggregated search, such as predicting which verticals to include on the main SERP in response to a user's query (vertical selection [9]) and predicting where to display each selected vertical on the SERP (vertical presentation [50]). ${ }^{6}$

In addition to research on algorithmic solutions for aggregated search, studies have also investigated how people interact with aggregated search interfaces. Two findings from prior work are important to our study. First, prior work has found that users are drawn to vertical results that are visually salient (e.g., images, videos). In a controlled laboratory setting, Sushmita et al. found a click bias in favor of video results [52] and image results [53]. In a production setting, prior work has also found that users have an attention bias toward vertical results (compared to web results) as measured by clicks [17, 42, 55] and mouseover events [20].

Vertical salience and attention bias play an important role in a second relevant finding from prior work. Prior studies have found that users do not always treat results from different verticals as being separate and independent. In other words, in an aggregated search environment, results from one source on the SERP may influence user engagement with results from other sources. Prior work has referred to this as a "spillover effect" $[5-8,13]$. Prior studies has found that the spillover effect can impact users' decisions to interact or not interact with a particular SERP. For example, a set of non-relevant image results on a SERP can influence users to assume that the web results are also non-relevant, even though the images and web results originate from independent back-end systems. Prior work has also found that different factors can influence the level of spillover from one group of results to another. Specifically, prior work has found that the level of spillover is stronger for verticals that are visually salient [5-8], verticals displayed higher on the SERP [6, 7], and verticals displayed without visual cues to distinguish them from other results (e.g., without a border or different-colored background) [6,7].

\footnotetext{
${ }^{5}$ As described in Section 3.5, we used the Stroop test to measure inhibition.

${ }^{6}$ Most of this research has assumed that results from the same vertical should be presented as a group.
} 


\subsection{Gestalt Principles}

Gestalt principles are a useful framework for understanding how people interact with aggregated search interfaces. Early work in psychology developed the Gestalt principles of pattern recognition, which help explain how people perceive or infer relations between items in an information display [38]. For example, the Gestalt principles of proximity, similarity, and common region state that items displayed close together, with a similar appearance, and with a common background (e.g., enclosed in a border) are perceived as a group [38, 48].

Prior work has found that interfaces designed based on Gestalt principles can allow users to be more effective and efficient. Outside of IR, Nygren [45] experimented with different website designs and found that participants were faster at findings items on pages where related items were grouped together. More closely related to IR, Dumais et al. [21] evaluated six different search interfaces that augmented search results with topical categories. Three interfaces were grouped (results from the same category were clustered together), and three interfaces were ungrouped. Participants completed search tasks faster with all three grouped interfaces.

As described further in Section 3.3, we used the Gestalt principles of proximity, similarity, and common region to design two aggregated search interfaces that presented the same results but did so in different ways. Compared to the interleaved interface, the blocked interface used the principles of proximity, similarity, and common region to more clearly distinguish between results from different sources (the web search engine and four different verticals).

\section{USER STUDY AND METHODS}

To investigate our three research questions, we conducted a laboratory study with 32 participants (15 female and 17 male). Participants were recruited using an opt-in mailing list of employees at our university. ${ }^{7}$ Participants were enrolled in the study based on the order in which they responded to our email advertisement. Participants were not screened in any way (e.g., using psychometric tests). Fourteen participants were student employees, and 18 participants were non-student employees. Participants' ages ranged from 19 to 55 years old (mean $=30.3$, median $=28.5$ ). Participants were asked to rate their search ability on a 7-point scale from "novice" (1) to "professional" (7). Overall, participants reported high levels of search ability (mean $=5.7$, median $=6$ ). Only four participants rated their search ability at a level of four or less.

\subsection{Study Protocol}

During the experiment, participants were first given an overview of the study and were asked to sign a consent form. ${ }^{8}$ Then, after completing a demographics questionnaire, participants completed four search tasks (Section 3.2). During each search task, participants were provided with a custom-built search system that returned results using five APIs: the Bing Web, News, Images, and Video Search APIs, ${ }^{9}$ and the eBay Product Search API. ${ }^{10}$

As described in Section 3.2, we designed four search tasks for participants to complete. In this article, we were not interested in studying task effects. Thus, to keep our tasks as consistent as possible, all tasks were comparative tasks. A comparative task requires comparing a set of items along a set of dimensions. For example, one of our tasks asked participants to compare three different water purification methods (boiling water, tablets, and portable water filters) along three dimensions

\footnotetext{
${ }^{7}$ Anyone on our university's payroll (students and non-students) can sign-up to this mailing list to receive emails about studies seeking participants. To help obtain a heterogeneous sample of participants, we enrolled both student and nonstudent employees.

${ }^{8}$ The study was reviewed by our University's Institutional Review Board (IRB).

${ }^{9}$ https://azure.microsoft.com/en-us/services/cognitive-services/.

${ }^{10} \mathrm{https} / / /$ go.developer.ebay.com/.
} 
(types of bacteria eliminated, cost, and how the method works). Participants were asked to search and bookmark at least 10 pages that would help them construct a response for the task. Participants were asked to provide a justification for each bookmark. We provided participants with a browser toolbar button to bookmark pages and enter their justifications in a pop-up form.

We experimented with two different aggregated search interfaces, referred to as the interleaved and blocked interfaces (Section 3.3). Participants completed the first two search tasks with one interface and the last two with the other. Participants completed two post-task questionnaires after each search task (Section 3.4). After finishing all four search tasks, participants took a short break and then completed three psychometric tests to measure their working memory, perceptual speed, and inhibition control (Section 3.5). We used a Latin square to rotate the ordering of our four search tasks and also balanced the interface order-half the participants completed the first two tasks with the interleaved interface, and the other half completed the first two tasks with the blocked interface. Participants were given 15 minutes to complete each search task and were given a 2-minute warning by the study moderator. The study session took about 1.5 hours, and participants were given US\$30 for participating in the study.

\subsection{Search Tasks}

One of our goals was to keep all four search tasks as consistent as possible. To this end, we designed four comparative tasks to use in the study. As previously mentioned, a comparative task is one that requires comparing a set of items along a set of dimensions. Our four tasks asked participants to compare three specific items along three specific dimensions. Comparative tasks have been used in prior IIR studies [15, 16]. Within the cognitive complexity framework [35], comparative tasks belong to the analyze task complexity category (i.e., an intermediate complexity level). The following is an example of one of our tasks:

"You are planning an extended hiking trip with your friend for this fall. You've recently heard that it can be unsafe to drink water directly from streams and rivers due to the possibility of contamination from bacteria, parasites, and pollutants. Now, you are trying to do some research on methods for purifying naturally-sourced water. Consider the following water purification methods: (1) boiling water (2) water purification tablets (3) using a portable water filter. For this task, your goal is to compare and contrast these methods based on the following criteria: (1) the types of bacteria eliminated by the method, (2) how much the method costs, and (3) how the method works."

Table 1 shows that topics, items, and dimensions associated with our four tasks.

We did not design our tasks to strictly require results from specific verticals. However, one could imagine video results being useful for Task 1 (heavy metal style and tempo), news results being useful for Tasks 2 (upcoming events), shopping results being useful for Task 3 (cost), and images being useful for Task 4 (typical ingredients).

As it turns out, our participants mostly used web results (i.e., versus vertical results such as images, news, shopping, and video results) to complete our tasks. To illustrate, on average, participants clicked on $13.773(\mathrm{SD}=5.085)$ web results and bookmarked $9.406(\mathrm{SD}=2.987)$ web results per session. In contrast, participants clicked on only $0.719(\mathrm{SD}=1.242)$ vertical results and bookmarked only $0.289(\mathrm{SD}=0.577)$ vertical results per session.

\subsection{Aggregated Search Interfaces}

As previously mentioned, participants were exposed to two interface conditions. Participants completed the first two tasks with one interface and the last two tasks with the other. We refer to one interface as the interleaved interface and the other as the blocked interface (Figures 2(a) and (b)). 
Table 1. Topics, Items, and Dimensions Associated with Our Four Tasks

\begin{tabular}{|c|c|c|c|}
\hline Task \# & Task Topic & Items & Dimensions \\
\hline \multirow{3}{*}{1} & \multirow{3}{*}{ heavy metal } & doom metal & origin \\
\hline & & thrash metal & style and tempo \\
\hline & & progressive metal & popular bands \\
\hline \multirow{3}{*}{2} & \multirow{3}{*}{ vacation city destination } & Miami, FL & weather in December \\
\hline & & San Diego, CA & attractions \\
\hline & & Santa Fe, NM & upcoming events \\
\hline \multirow{3}{*}{3} & \multirow{3}{*}{ water purification methods } & boiling water & bacteria eliminated \\
\hline & & purification tablets & cost \\
\hline & & water filter & how the method words \\
\hline \multirow{3}{*}{4} & \multirow{3}{*}{ Chinese cuisines } & Cantonese & region of origin \\
\hline & & Shandong & typical ingredients \\
\hline & & Sichuan & popularity in the U.S. \\
\hline
\end{tabular}

Both interfaces had several common characteristics. First, both systems integrated results from five different sources on the default aggregated SERP: web, image, news, video, and shopping results. We used Bing APIs to return web, image, news, and video results and the eBay API to return shopping results. The main aggregated SERP displayed the top- 10 web results, top- 5 images results, top-3 news results, top-5 video results, and top-5 shopping results. Given the same query, both systems displayed the same results. Second, in both systems, results were displayed using the same surrogate representations. Web results were displayed using the title, summary snippet and URL; image results were displayed using thumbnails; news results were displayed using the article's title, summary snippet, news source, publication age, and thumbnail; shopping results were displayed using the product's title, price, condition, and thumbnail; and video results were displayed using the video's title, duration, and thumbnail. Finally, both systems provided tabs that allowed participants to view results from a specific source for the current query and to issue new queries. Within each source-specific tab, we provided pagination controls and presented 10 results per page.

Interleaved Interface: With the interleaved interface (Figure 2(a)), our goal was to study participants' search behaviors with an aggregated search interface that does not clearly distinguish between results from different sources on the main SERP. The interleaved interface displayed results using the following rules. First, the top-5 image results were always displayed at the top. Second, after each top-10 web result, we randomly interleaved one top-ranked news, shopping, or video result. Finally, we interleaved the remaining news, shopping, and video results at the bottom of the SERP in a pseudo-random fashion by randomly picking a vertical and interleaving its top-most result (not already interleaved). ${ }^{11}$

Blocked Interface: With the blocked interface (Figure 2(b)), our goal was to study participants' search behaviors with an aggregated search interface that clearly distinguishes between results from different sources on the main SERP. The blocked interface displayed results using a two-column format, and results from the same source were always displayed as a group and always in the same region on the SERP. The top-5 image results were displayed horizontally in the top-left region, the top-10 web results were displayed vertically in the middle-left region, the top-3 news results were displayed vertically in the top-right region, the top- 5 video results were

\footnotetext{
${ }^{11}$ We decided to show the top-five image results as a group (rather than vertically interleaved with the other results) to keep the "look and feel" of the interleaved interface somewhat consistent with commercial search portals (e.g., Google).
} 


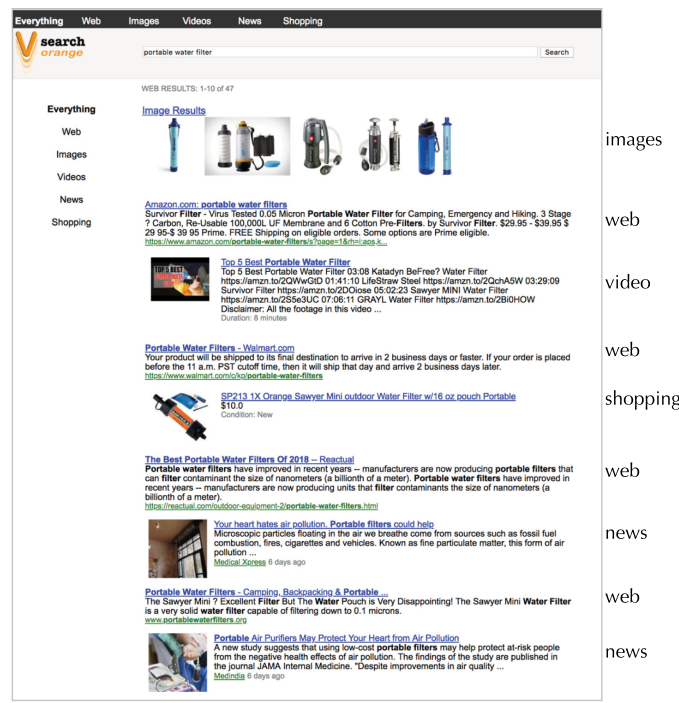

(a) Interleaved Interface (truncated)

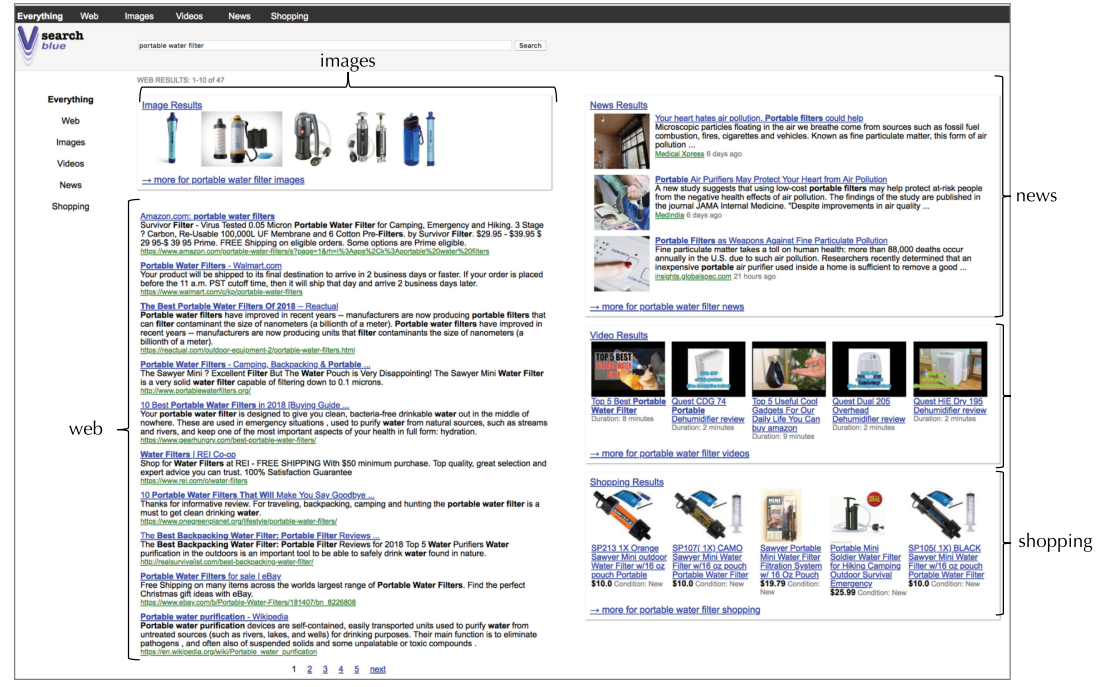

(b) Blocked Interface

Fig. 2. Aggregated search interfaces.

displayed horizontally in the middle-right region, and the top-5 shopping results were displayed horizontally in the bottom-right region. Furthermore, the image, news, shopping, and video results were enclosed in a border with a shadow effect to help distinguish them from each other and the web results.

With respect to the Gestalt Principles discussed Section 2.5, the blocked interface used three Gestalt principles to help distinguish between results from different sources. In terms of proximity, results from the same source were displayed as a group and always in the same region on the SERP. In terms of similarity, each group of results was displayed according to its own unique surrogate representation. Finally, in terms of common region, the border around the image, news, shopping, 
Table 2. NASA TLX Questionnaire to Measure Workload

\begin{tabular}{l}
\hline Mental demand: How mentally demanding was the task? \\
\hline Physical demand: How physically demanding was the task? \\
\hline Temporal demand: How hurried or rushed was the pace of the task? \\
\hline Failure: How successful were you in accomplishing what you were asked to do? \\
\hline Effort: How hard did you have to work to accomplish your level of performance? \\
\hline Frustration: How insecure, discouraged, irritated, stressed, annoyed were you? \\
\hline \hline
\end{tabular}

Table 3. User Engagement Scale (Short Form)

\begin{tabular}{l}
\hline FA1: I lost myself in this search experience. \\
\hline FA2: The time I spent searching just slipped away. \\
\hline FA3: I was absorbed in the search task. \\
\hline PU1: I felt frustrated while doing the search task. (reverse coded) \\
\hline PU2: My search experience was taxing. (reverse coded) \\
PU3: I found the search system confusing to use. (reverse coded) \\
AE1: The search system was attractive. \\
AE2: The search system was aesthetically appealing. \\
AE3: The search system appealed to my senses. \\
RW1: My search experience was worthwhile. \\
\hline RW2: My search experience was rewarding. \\
\hline \hline RW3: I felt interested in the search task.
\end{tabular}

and video results provided an additional visual cue to help distinguish them from each other and the web results.

\subsection{Post-task Questionnaires}

After finishing each search task, participants completed two questionnaires aimed to measure workload and user engagement. To measure workload, participants were asked six questions from the raw NASA TLX [30] questionnaire (Table 2). These six questions are designed to measure: (1) mental demand, (2) physical demand, (3) temporal demand, (4) failure, (5) effort, and (6) frustration. Participants responded to all NASA TLX questions using a 7-point scale with labeled endpoints. For the failure question, the labeled end-points were "perfect" (1) and "failure" (7). For the other five questions, the labeled end-points were "very low" (1) and "very high" (7). Thus, for all NASA TLX questions, high response values indicate high levels of workload. ${ }^{12}$

To measure user engagement, we used a short-form of O'Brien's User Engagement Scale [46], denoted as UES-SF. The UES-SF questionnaire (Table 3) contains 12 items designed to capture 4 factors of engagement (3 items per factor): (1) focused attention (FA), (2) perceived usability (PU), (3) aesthetic appeal (EA), and (4) reward (RW). Participants indicated their level of agreement with all 12 UES-SF items using a 7-point scale with labeled endpoints, from "strongly disagree" (1) to "strongly agree" (7). We examined the internal consistency of each three-item factor using Cronbach's alpha and found acceptable values: focused attention $=0.793$, perceived usability $=0.770$, aesthetic appeal $=0.945$, and reward $=0.863$. As shown in Table 3, the three items for perceived

\footnotetext{
${ }^{12}$ In the original NASA TLX instrument, participants mark their responses using a 20-point scale. In our case, we used a 7-point scale. Thus, our results are not directly comparable with previous results that used the original 20-point scale.
} 
usability were reverse coded. Thus, in our results, high response values for each user engagement factor indicate high levels of engagement.

\subsection{Cognitive Ability Tests}

After completing all four search tasks, participants completed three psychometric tests to measure their working memory, perceptual speed, and inhibition. The order of the tests was rotated across participants. The instructions for each psychometric test were read out loud while the participant followed along with a printed sheet of paper.

Perceptual Speed: To measure perceptual speed, we used the Finding A's test provided by the Ekstrom Kit of Factor-referenced Cognitive Tests [22]. During the Finding A's test, participants complete a series of 2-minute trials in which they are given lists of words and must draw a line through all words containing the letter "A." Participants are instructed to work quickly without sacrificing accuracy. The final score is equal to the number of words with the letter A found by the participant (analogous to recall). A high score indicates that the participant was able to scan words quickly without sacrificing accuracy. The Finding A's test has been used in prior IIR studies to measure perceptual speed [14, 54].

Working Memory: To measure working memory, we used a computerized memory span test distributed with the "CogLab in a CD" textbook by Francis et al. [25]. During this test, participants complete a series of trials. During each trial, the participant is displayed a sequence of $N$ items one at a time. After this, the participant is shown a set of nine items (in a grid display) and is asked to recall the items that appeared in the sequence in the same order. If the participant answers correctly, then the number of items in the next sequence is increased by one (up to a maximum of nine). Otherwise, the number of items in the next sequence is decreased by one. The test shows participants five different types of items: digits, letters that sound similar, letters that sound dissimilar, short words, and long words. Finally, the test outputs a score for each item type. Each score corresponds to the length of the last sequence answered correctly for that item type. To compute a final score, we averaged participants' scores across all five item types. The same memory span test has been used in prior IIR studies to measure working memory ability [27, 29].

Inhibition: To measure inhibition, we used the Stroop test, which is also a computerized test. Again, we used the version of the Stroop test distributed with the "CogLab in a CD" textbook by Francis et al. [25]. During the Stroop test, participants are flashed a sequence of words indicating one of three colors: red, green, or blue. For each word, the participant must quickly indicate the color of the word by pressing a specific key (e.g., the "B" key for the color blue). Some of the words displayed are congruent (e.g., the word "blue" displayed in blue font), and others are incongruent (e.g., the word "blue" displayed in red font). Participants must complete 48 correct trials, of which 24 are congruent and 24 are incongruent. The final score is equal to the participant's average response time (in milliseconds) for the incongruent trials minus the average response time for the congruent trials. Response times are typically slower for the incongruent trials, an effect referred to as the Stroop effect. For the Stroop test, lower scores are better. Higher scores indicate greater difficulty in focusing on the relevant stimulus (the color of the word) and ignoring (or inhibiting attention to) the non-relevant stimulus (the word itself).

The Stroop test was first introduced in 1935 [51], and there are many variants (see MacLoed [41]). As noted in Kane and Engle [34], the Stroop test is a "mainstay of research concerning selective attention and the external versus executive control of behavior" (p. 48). Research outside of IR has used the Stroop test to measure inhibitory attention control [26]. To our knowledge, the Stroop test has not been used in IR studies to measure individuals' inhibitory attention control. As described in Section 2, Gwidska [28] used Stroop-like tests (as secondary tasks) to measure workload while participants completed search tasks. 


\subsection{Search Behaviors}

Behavioral Measures: To investigate RQ3, we examined the effects of working memory, perceptual speed, and inhibition on participants' search behaviors. To this end, we logged participants' interactions with both interfaces, including their queries, clicks, bookmarks, mouseover events, and scroll events. Using these logged data, we generated the following 15 behavioral measures. Below, we report on a factor analysis that helps explain the underlying phenomena being captured by these measures.

(1) Queries: number of queries in the session.

(2) Clicks: number of SERP clicks in the session.

(3) Bookmarks: number of bookmarks in the session.

(4) Completion time: session length (in seconds).

(5) Time to first bookmark: time taken to produce the first bookmark (in seconds).

(6) Query-to-click time: avg. time (in seconds) between each query and its first SERP click, if any.

(7) Click-to-bookmark time: for each bookmark, average time (in seconds) between the SERP click and the bookmark.

(8) Time between events: avg. time (in seconds) between subsequent search actions of the following types: queries, clicks, and bookmarks.

(9) Clicks without a bookmark: number of SERP clicks that did not yield a bookmark.

(10) Queries without clicks: number of abandoned queries (i.e., without at least one SERP click).

(11) Queries without mouseovers: number of queries without at least one mouseover event.

(12) Queries without scrolls: number of queries without a scroll event.

(13) Queries without bookmarks: number of queries without a bookmarked page.

(14) Quick query reformulations: number of queries issued within 60s of the previous query. This measure aimed to capture query abandonment or queries for which the participant did not engage deeply with the search results.

(15) Queries with repeated intent: number of queries with the same search intent as a previous query (explained below).

Queries with a Repeated Intent: The last measure above requires more explanation. To compute the number of queries with a repeated intent, we performed a qualitative analysis of all queries issued by participants. As previously mentioned, our tasks required participants to compare/contrast three items along three dimensions. We manually coded participants' queries based on the item(s) and dimension(s) mentioned in the query (i.e., a two-dimensional coding scheme). The item(s) and dimension(s) were indexed based on their order of appearance during the search session.

To illustrate, Table 4 shows the first five queries from a participant's query stream and their respective item and dimension codes. The first query is coded as $\left({ }^{* *}, *\right)$, because it mentions no items or dimensions. The second query is coded as $\left(1,{ }^{*}\right)$, because it mentions the first item in the query stream and no dimension. The third query is coded as $\left({ }^{*}, 1\right)$, because it mentions the first dimension in the query stream and no item. The fourth query is coded as $(2,2)$, because it mentions the second item and second dimension in the query stream. Finally, the fifth query is also coded as $(2,2)$, because it mentions the second item and dimension in the query stream. The fifth query would be considered one with a repeated search intent (same as the fourth query). 
Table 4. Examples of Item and Dimension Codes for First Five Queries in a Stream

\begin{tabular}{r|cc}
\hline Query & Item Code & Dimension Code \\
\hline best water purification method hiking & $*$ & $*$ \\
portable water filter & 1 & $*$ \\
cost comparison water purification while hiking & $*$ & 1 \\
bacteria killed by boiling water & 2 & 2 \\
boiling water microorganisms eliminated & 2 & 2 \\
\hline
\end{tabular}

To test the reliability of our coding scheme, both authors coded the query streams from four participants (12.5\% of all query streams). With respect to the item codes, the Cohen's Kappa agreement was $\kappa=0.986$. With respect to the dimension codes, the Cohen's Kappa agreement was $\kappa=0.951$. Both levels of agreement were at the level of "near perfect agreement" [39]. Given this high level of agreement, one of the authors coded the remaining query streams.

Aggregated vs. Vertical SERP Interactions: In both the interleaved and blocked interfaces, the default SERP was an aggregated SERP. However, similar to commercial portals such as Google, both interfaces allowed participants to use tabs to exit the default SERP and navigate to a specific vertical. Participants could use these tabs to see only results from a specific vertical (e.g., only images) for the current query and/or to issue new queries (all verticals had a query box). ${ }^{13}$ As shown in Figures 2(a) and 2(b), these vertical tabs were provided along the top and left side of the interface.

As it turns out, while using both the interleaved and blocked interfaces, participants rarely used these tabs and interacted mostly with the default aggregated SERP. To illustrate, participants produced a total of 1,855 SERP clicks and only 65 of these were on a vertical SERP. In fact, there were only 19 (of 128) search sessions in which the participant clicked on at least one tab to exit the default aggregated SERP. Given this low level of interaction with vertical SERPs, our 15 behavioral measures for RQ3 focused on interactions with any SERP, which was overwhelmingly the aggregated SERP.

Factor Analysis: To better understand the underlying phenomena captured by our 15 behavioral measures, we conducted an exploratory factor analysis using all 128 search sessions (32 participants $\times 4$ sessions per participant). We conducted a principal component analysis (with varimax rotation) and found a solution with three factors that explained $68 \%$ of the variance. Table 5 shows the final factor loading matrix. Note that three measures (the number of quick query reformulations, clicks, and the task completion time) had high loadings for more than one factor.

We interpreted the three factors as follows. Factor 1 is related to the extent to which participants issued queries where they did not engage with the search results provided (i.e., had difficulty identifying relevant results on SERPs). The measures with the greatest loadings with Factor 1 were as follows: number of queries; number of queries without bookmarks, clicks, scrolls, or mouseovers; number of quick query reformulations; and number of queries with a repeated intent (same as previously issued query). Factor 2 is related to the participant's pace of interaction (e.g., time between events, time to first bookmark, click-to-bookmark time, query-to-click time, completion time, etc.). Factor 2 had three measures with high negative loadings. These negative loadings suggests that participants who interacted at a slower pace had fewer quick query reformulations, clicks, and bookmarks. Finally, Factor 3 is related to the extent to which participants clicked on results that did not yield a bookmarked page (i.e., abandoned clicks). Based on the three measures with high

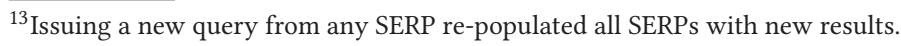


Table 5. Factor Analysis of Behavioral Measures

\begin{tabular}{l|lll}
\hline & Factor 1 & Factor 2 & Factor 3 \\
\hline queries without bookmarks & 0.888 & 0.021 & 0.216 \\
queries with repeated intent & 0.867 & 0.029 & 0.217 \\
queries & 0.863 & -0.285 & 0.214 \\
queries without clicks & 0.863 & 0.232 & 0.029 \\
quick query reformulations & 0.811 & -0.412 & -0.083 \\
queries without mouseovers & 0.696 & 0.118 & -0.018 \\
queries without scrolls & 0.577 & -0.370 & -0.004 \\
time between events & -0.259 & 0.887 & -0.027 \\
time to first bookmark & 0.019 & 0.718 & 0.202 \\
bookmarks & -0.045 & -0.659 & 0.242 \\
click-to-bookmark time & -0.207 & 0.626 & -0.072 \\
query-to-click time & 0.134 & 0.523 & -0.065 \\
clicks & 0.143 & -0.462 & 0.851 \\
clicks without bookmarks & 0.161 & -0.033 & 0.835 \\
completion time & 0.045 & 0.577 & 0.674 \\
\hline
\end{tabular}

The dark gray cells indicate the strongest (positive or negative) loading of each behavioral measures onto a factor. The light gray cells indicate factors loadings that were not the strongest but worth pointing out (i.e., $>|0.40|$ ). Behavioral measures with high loadings onto more than one factor can be interpreted as being somewhat "ambiguous." For example, users may quickly reformulate queries, because they are having difficulty finding relevant results on SERPs (Factor 1) or because they are generally interacting at a faster pace (Factor 2).

Table 6. Descriptive Statistics for Scores Associated with Each Cognitive Ability Test

\begin{tabular}{llll}
\hline & Working Memory & Perceptual Speed & Inhibition \\
\hline Possible Range & $0-9$ & $0-200$ & unbounded \\
Mean (SD) & $4.86(0.79)$ & $64.16(12.00)$ & $107.80(166.27)$ \\
Median & 5.60 & 63 & 101.80 \\
Min, Max & $3.80,7.20$ & 44,90 & $-272.20,449.13$ \\
Participants in Low Group & 19 & 17 & 16 \\
Participants in High Group & 13 & 15 & 16 \\
\hline
\end{tabular}

loadings with Factor 3, participants with more abandoned clicks had more clicks in general and took longer to complete the task. This factor analysis will help in understanding our RQ3 results.

\subsection{Data Analysis}

In this section, we report on our participants' cognitive ability test scores and explain two important decisions made during data analysis: (1) binning participants into high and low groups for each cognitive ability and (2) using multilevel modeling for our statistical analyses.

Cognitive Ability Test Scores: Table 6 shows descriptive statistics associated with participants' scores for all three cognitive ability tests. To better understand these scores, we compared them against a few reference scores. In terms of perceptual speed, Turpin et al. [54] administered the Finding A's test to 16 study participants from the same population as the participants in our study (staff from the same university). Participants in that study had a mean score of 51.94 $(\mathrm{SD}=10.41)$. In terms of working memory, Gwizdka [29] administered the same computerized 
working memory test to 26 undergraduate students (a slightly different population than our participants). Gwizdka scored participants using their average score for two of the five item-types associated with the working memory test: long and short words. ${ }^{14}$ In Gwizdka's study, participants had a mean score of $5.45(\mathrm{SD}=0.72)$. In our study, for the same two item-types, participants had a mean score of $4.86(\mathrm{SD}=0.79)$. Finally, in terms of inhibition, Cothran and Larsen [18] administered the Stroop test to 32 undergraduate students (again, a slightly different population than our participants). Participants had a mean score of $114(\mathrm{SD}=18.85) .{ }^{15}$ For all three tests, our participants' mean scores were within one standard deviation of the reference score.

To analyze the effects of each cognitive ability, we decided to group participants into low and high cognitive ability groups using a median split. Table 6 shows the number of participants in each group for each cognitive ability. For working memory, participants were divided at 5.60 (low $=3.80-5.60$, high $=5.61-7.20)$. For perceptual speed, participants were divided at 63 (low $=$ 44-63, high $=64-90)$. For inhibition, participants were divided at 101.80 (low $=101.80-449.13$, high $=-272.20-101.79)$. Note that for inhibition, low test scores indicate high ability. Prior studies have also analyzed the effects of different cognitive abilities by binning participants into low/high groups using either a median split [1, 14, 54] or by taking the bottom and top quartiles [29].

We also examined the Pearson's correlation values between participants' scores for all pairs of cognitive ability tests. None of the correlations were statistically significant. The correlation between working memory and perceptual speed was $r=0.069(p=0.707)$, the correlation between working memory and inhibition was $r=0.133(p=0.469)$, and the correlation between perceptual speed and inhibition was $r=0.165(p=0.366)$. These non-significant correlation values suggest that the three cognitive ability tests given to participants measured different cognitive abilities. After binning participants into high/low working memory (WM), perceptual speed (PS), and inhibition (IN) groups, there were only 7 (of 32) participants assigned to both the high-WM and high-PS groups, 7 participants assigned to both the high-WM and high-IN groups, 6 participants assigned to both the high-PS and high-IN groups, and 2 participants assigned to all three high-ability groups.

Statistical Analysis: To investigate our three research questions, we were interested in measuring the following main and interaction effects on each outcome: (1) the main effect of the interface condition (1 main effect), (2) the main effect of each cognitive ability (3 main effects), and (3) the two-way interaction effects between the interface condition and each cognitive ability (3 two-way interaction effects). In our statistical analyses, we decided to use multilevel modeling.

Multilevel modeling (MLM) is similar to other regression analysis techniques in which there are several predictor variables and one outcome variable. However, MLM is particularly powerful when the data are "grouped," and we wish to account for random effects introduced by different groups. In our case, each of our 32 participants completed each of the 4 search tasks described in Section 3.2. In this respect, our dataset consisted of 128 data points $(32 \times 4)$ that were grouped along two dimensions: participant and task. In our modeling, we wanted to account for random effects at the participant level and at the task level. At the participant level, random effects might be due to differences between participants beyond the three cognitive abilities examined (e.g., search experience, domain expertise, etc.). At the task level, while all our tasks were comparative tasks, random effects might be due to differences beyond task-type (e.g., one topical domain being more difficult than another). To model random effects at the participant and task level, we used participant and task as random-intercept factors in all our models. This model configuration is

\footnotetext{
${ }^{14}$ Gwizdka motivated this decision based on the notion that search is more greatly impacted by the ability to remember words than letters and digits.

${ }^{15}$ Note that this standard deviation is smaller than the standard deviation of our participants' scores (i.e., 166.27). This may be because our participants had a wider age range.
} 
referred to as a mixed-effects model with crossed random effects (for more details, see Baayen and Bates [11]).

Each of our models included seven predictors (i.e., fixed factors): one for interface condition, one for working memory group, one for perceptual speed group, one for inhibition group, and one for each two-way interaction between interface condition and each cognitive ability group. For each cognitive ability variable, the low group was coded as "0" and the high group was coded as "1." Thus, positive $\beta$ values indicate greater values of the outcome variable for the high cognitive ability group. For the interface condition, the interleaved interface was coded as " 0 " and the blocked interface was coded as " 1 ." Thus, positive $\beta$ values indicate greater values of the outcome variable for the blocked interface. ${ }^{16}$

To further explain, each of our models used the following equation:

$$
Y_{i j k}=\underbrace{\beta_{0}+\mu_{j}+\mu_{k}}_{\text {random intercept }}+\underbrace{\beta_{1} X_{1 i}+\beta_{2} X_{2 i}+\cdots+\beta_{7} X_{7 i}}_{\text {fixed factors }}+\underbrace{e_{i j k}}_{\text {random error }},
$$

where $Y_{i j k}$ denotes the outcome variable for data point $i$, which is associated with participant $j$ and task $k$. As shown, the y-intercept in our models was a linear combination of three parameters: $\beta_{0}$ (a global parameter), $\mu_{j}$ (specific to participant $j$ ), and $\mu_{k}$ (specific to task $k$ ). Parameters $\beta_{1} \ldots \beta_{7}$ denote the $\beta$ values associated with our seven fixed factors (four main effects and three interaction effects).

In the next sections, we report on our results with respect to research questions RQ1-RQ3. Summaries of all mixed-effects models are provided in Appendix A.

\section{RQ1 RESULTS: EFFECTS ON WORKLOAD}

In our first research question (RQ1), we investigate the effects of the interface condition and each cognitive ability on the levels of workload reported by participants. To address this question, we analyzed participants' responses to six items from the NASA-TLX questionnaire: (1) mental demand, (2) physical demand, (3) temporal demand, (4) failure, (5) effort, and (6) frustration. Additionally, we averaged responses to these items to measure overall workload. Figure 3 shows the means and 95\% confidence intervals for each workload measure according to interface condition and each cognitive ability: working memory (Figure 3(a)), perceptual speed (Figure 3(b)), and inhibition (Figure 3(c)).

In all figures for RQ1-RQ3, significant differences between low/high cognitive ability groups can be seen by focusing on columns labeled "Both" (i.e., both interfaces). Similarly, significant interaction effects between low/high groups and the interface condition can be seen by comparing differences between low/high groups in columns labeled "Int." (i.e., interleaved) and "Block." (i.e., blocked). In cases of an interaction effect, the differences between low/high groups are larger (or different) with one interface versus the other.

Main Effects of Interface Condition: Interface condition did not have a significant main effect for any measure of workload.

Main Effects of Cognitive Abilities: Working memory had a significant main effect for two measures of workload (Figure 3(a)). Participants in the high-WM group reported significantly lower physical demand $(\beta=-0.757$, S.E. $=0.372, p=0.048)$ and frustration $(\beta=-1.239$, S.E. $=0.379, p=0.002)$ than participants in the low-WM group. Perceptual speed (Figure 3(b)) and inhibition (Figure 3(c)) did not have a significant main effect for any measure of workload.

\footnotetext{
${ }^{16}$ In total, we performed 27 regressions (one per outcome measure): 7 workload measures, 5 engagement measures, and 15 behavioral measures. Given that our study only included 128 data points ( 32 participants $\times 4$ tasks per participant), we decided to not apply corrections to our statistical tests.
} 


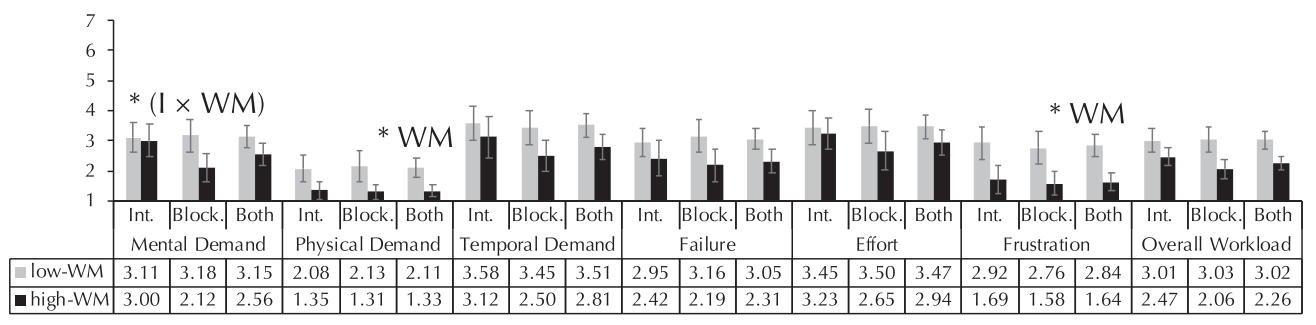

(a) Working Memory

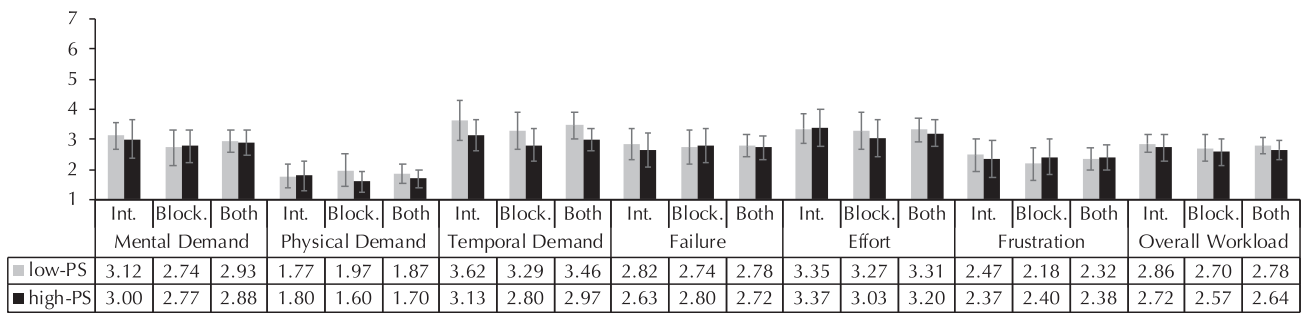

(b) Perceptual Speed

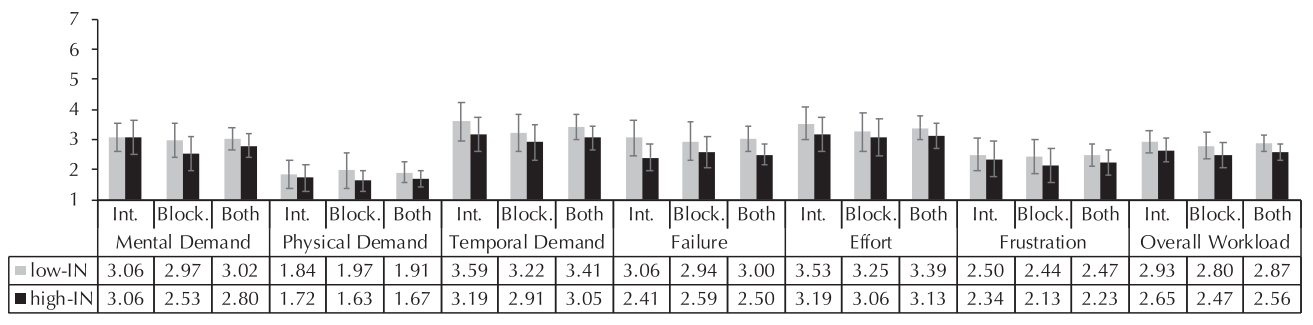

(c) Inhibition

Fig. 3. Post-task evaluations of workload according to interface and cognitive ability: working memory (WM), perceptual speed (PS), and inhibition (IN). Int.= interleaved interface, Block.= blocked interface. Symbol *WM denotes a significant main effect of working memory and symbol *(IXWM) denotes a significant interaction effect of interface and working memory $(p<0.05)$.

Interaction Effects: Interface condition and working memory had a significant interaction effect for mental demand $(\beta=-0.949$, S.E. $=0.425, p=0.028)$. As shown in Figure 3(a), with the interleaved interface, high- and low-WM participants reported comparable levels of mental demand. Conversely, with the blocked interface, high-WM participants reported significantly lower mental demand than low-WM participants. One possible explanation is that high-WM participants were more effective at keeping the fixed layout of the blocked interface in active memory. We elaborate on our interpretations of these results in Section 7.

\section{RQ2 RESULTS: EFFECTS ON USER ENGAGEMENT}

In our second research question (RQ2), we investigate the effects of the interface condition and each cognitive ability on the levels of user engagement reported by participants. To address this question, we analyzed participants' responses to the UES-SF questionnaire, which measures four factors of user engagement: (1) perceived usability, (2) aesthetic appeal, (3) focused attention, and (4) reward. Additionally, we averaged responses for these four factors to measure overall engagement. Figure 4 shows the means and $95 \%$ confidence intervals for each engagement measure 


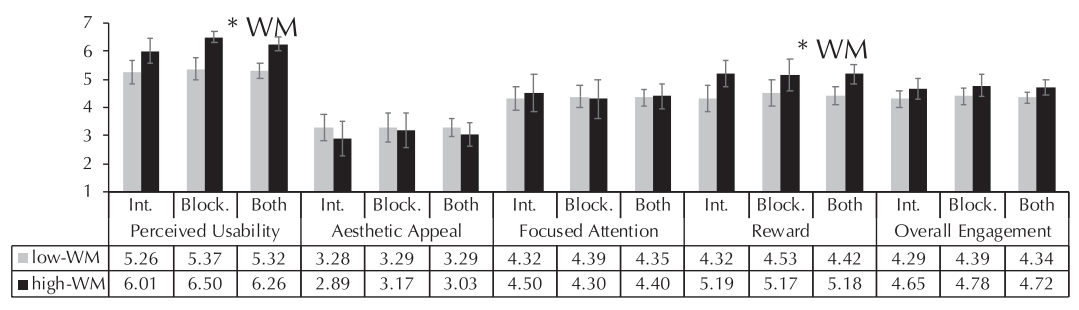

(a) Working Memory

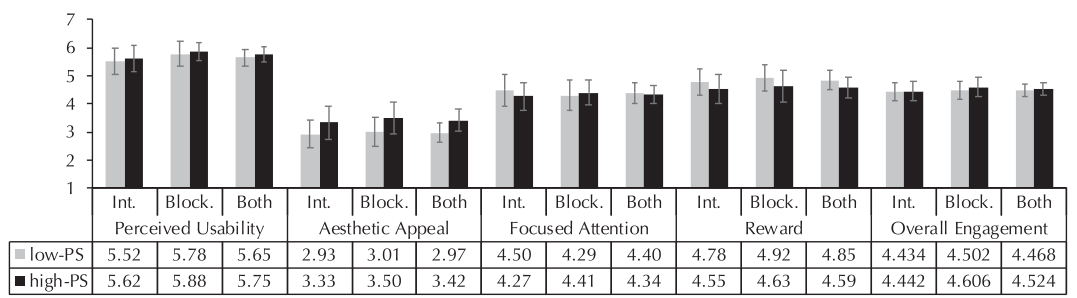

(b) Perceptual Speed

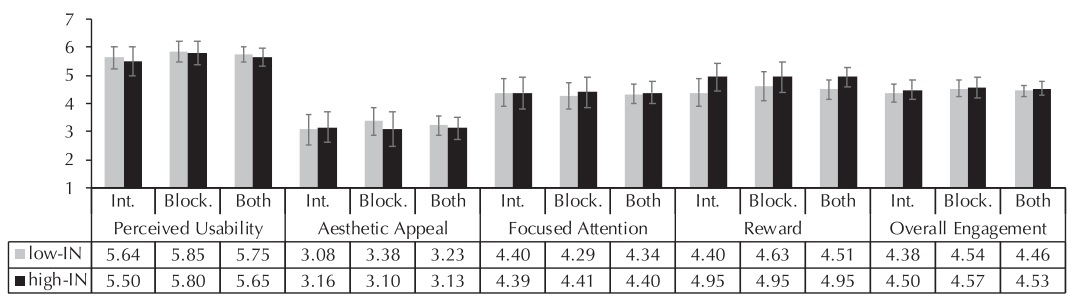

(c) Inhibition

Fig. 4. Post-task evaluations of user engagement according interface and cognitive ability: working memory (WM), perceptual speed (PS), and inhibition (IN). Int.= interleaved interface, Block.= blocked interface. Symbol *WM denotes a significant main effect of working memory $(p<0.05)$.

according to interface condition and each cognitive ability: working memory (Figure 4(a)), perceptual speed (Figure 4(b)), and inhibition (Figure 4(c)).

Main Effects of Interface Condition: Interface condition did not have a significant main effect for any measure of engagement.

Main Effects of Cognitive Abilities: Working memory had a significant main effect for two factors of user engagement (Figure 4(a)). Participants in the high-WM group reported significantly higher perceived usability $(\beta=0.736$, S.E. $=0.312, p=0.022)$ and reward $(\beta=1.013$, S.E. $=0.355$, $p=0.006)$ than participants in the low-WM group. Perceptual speed (Figure 4(b)) and inhibition (Figure 4(c)) did not have a significant main effect for any measure of engagement.

Interaction Effects: We did not observe any significant two-way interaction effects between the interface condition and each cognitive ability for any measure of engagement.

\section{RQ3 RESULTS: EFFECTS ON SEARCH BEHAVIORS}

In our third research question (RQ3), we investigate the effects of the interface condition and each cognitive ability on participants' search behaviors. To address this question, we analyzed the 15 behavioral measures described in Section 3.6. 


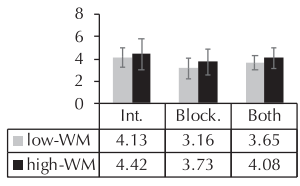

(a) Queries w/o bookmarks

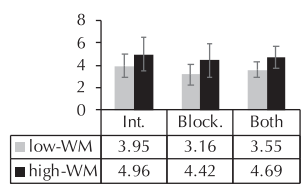

(e) Quick query reform.

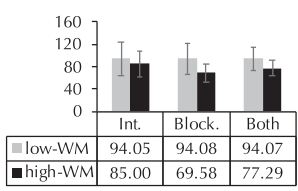

(i) Time to 1 st book

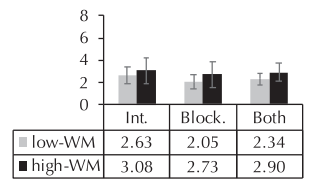

(b) Queries w/ repeat. intent

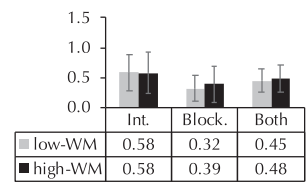

(f) Queries w/o mouseovers

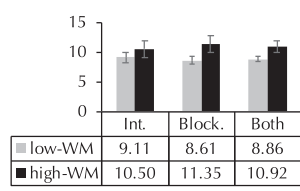

(j) Bookmarks

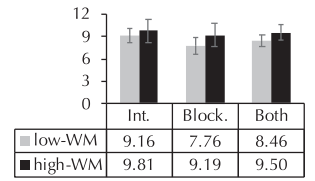

(c) Queries

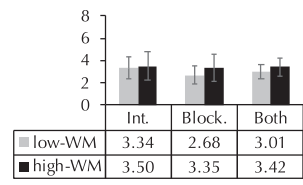

(g) Queries w/o scrolls

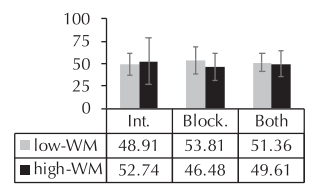

(k) Click-to-bookmark time

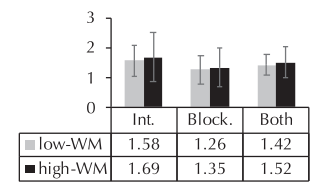

(d) Queries w/o clicks

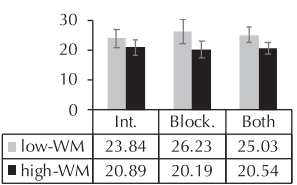

(h) Time b/w events

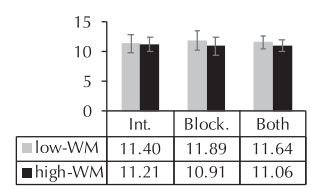

(I) Query-to-click time

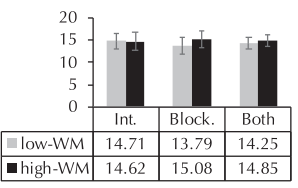

(m) Clicks

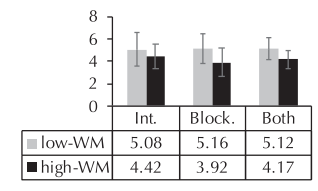

(n) Clicks w/o bookmarks

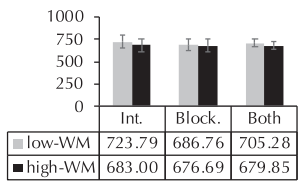

(o) Completion time

Fig. 5. Effects of working memory group (low-WM, high-WM), and interface (Int. = interleaved, Block. = blocked) on participants' search behaviors.

\subsection{Main Effects of Interface Condition}

Interface condition had a significant main effect on the number of queries issued by participants. Participants issued significantly more queries with the interleaved interface $(9.42 \pm 0.84)$ than with the blocked interface $(8.34 \pm 0.93)(\beta=-2.020$, S.E. $=0.947, p=0.036)$. Additionally, interface condition had a strong (but not significant) effect on the number of queries without any bookmarks. Participants issued more queries without any bookmarks with the interleaved interface $(4.25 \pm 0.74)$ than the blocked interface $(3.39 \pm 0.71)(\beta=-1.470$, S.E. $=0.761, p=0.056)$.

\subsection{Working Memory: Main and Interaction Effects}

Figure 5 shows the means and 95\% confidence intervals for our 15 behavioral measures according interface condition and working memory group. In Figures 5-7, the sub-figures are ordered consistent with Table 5. In other words, measures (a)-(g) are those related to Factor 1 (difficulty finding relevant SERP results), measures (h)-(l) are those related to Factor 2 (pace of interaction), and measures (m)-(o) are those related to Factor 3 (abandoned clicks).

Main Effects: Working memory did not have a significant effect on any of our 15 behavioral measures. Working memory had a strong (but not significant) effect on the number of bookmarks $(\beta=1.438$, S.E. $=0.750, p=0.060)$. High-WM participants produced more bookmarks than lowWM participants.

Interaction Effects: Working memory and interface condition did not have a significant interaction effect for any behavioral measure. 


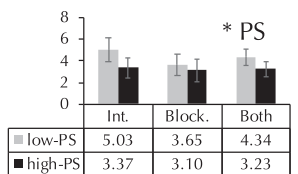

(a) Queries w/o bookmarks

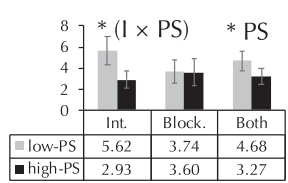

(e) Quick query reform

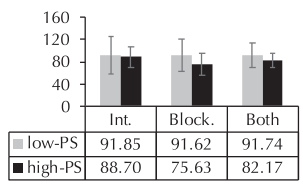

(i) Time to $1 \mathrm{st}$ book.

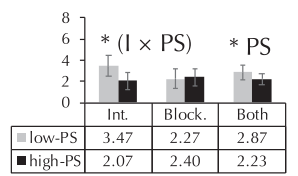

(b) Queries w/ repeat. intent

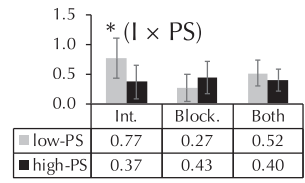

(f) Queries w/o mouseovers

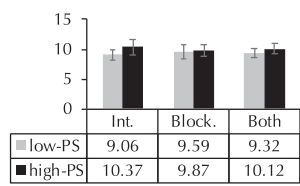

(j) Bookmarks

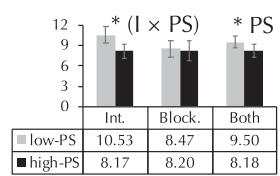

(c) Queries

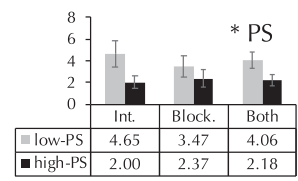

(g) Queries w/o scrolls

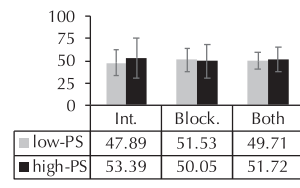

(k) Click-to-bookmark time

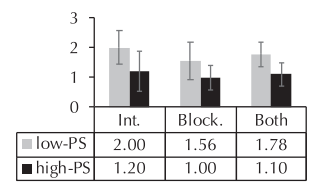

(d) Queries w/o clicks

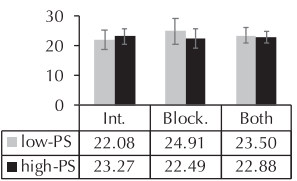

(h) Time b/w events

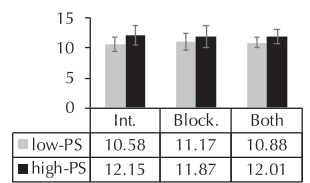

(I) Query-to-click time

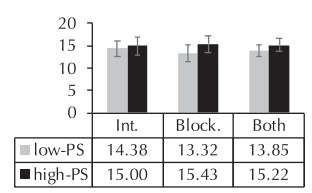

(m) Clicks

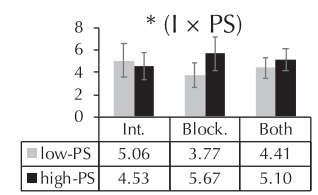

(n) Clicks w/o bookmarks

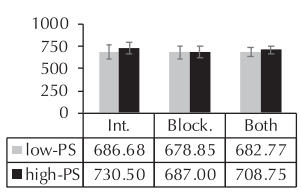

(o) Completion time

Fig. 6. Effects of perceptual speed group (low-PS, high-PS) and interface (Int. = interleaved, Block. = blocked) on participants' search behaviors. Symbol *PS denotes a significant main effect of perceptual speed group and symbol * (IXPS) denotes a significant interaction effect of interface and perceptual speed group $(p<$ $0.05)$.

Summary: Overall, we did not observe significant differences between low- and high-WM participants across our 15 behavioral measures. That said, the observed trend is that high-WM participants worked at a faster place (e.g., less time between events) and performed more search actions (e.g., more bookmarks) than low-WM participants.

\subsection{Perceptual Speed: Main and Interaction Effects}

Figure 6 shows the means and 95\% confidence intervals for our 15 behavioral measures according interface condition and perceptual speed group.

Main Effects: Perceptual speed had a significant main effect for five measures:

(1) queries without bookmarks $(\beta=-1.741$, S.E. $=0.790, p=0.032)$

(2) queries with repeated intent $(\beta=-1.430$, S.E. $=0.648, p=0.031)$

(3) queries $(\beta=-2.401$, S.E. $=0.926, p=0.012)$

(4) quick query reformulations $(\beta=-2.746$, S.E. $=0.837, p=0.002)$

(5) queries without scrolls $(\beta=-2.575$, S.E. $=0.662, p=0.000)$

All five measures were higher for low-PS participants than high-PS participants.

Interaction Effects: Perceptual speed and interface condition had a significant interaction effect for five measures: 


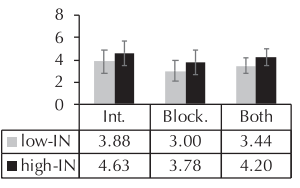

(a) Queries w/o bookmarks

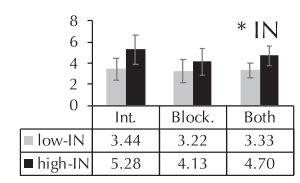

(e) Quick query reform.

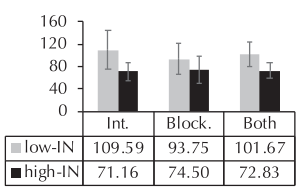

(i) Time to 1 st book.

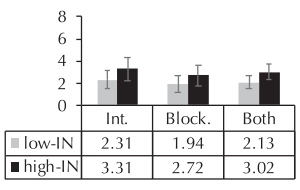

(b) Queries w/ repeat. intent

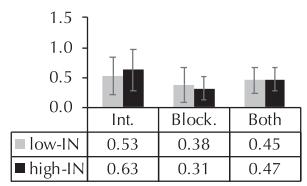

(f) Queries w/o mouseovers

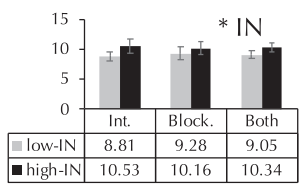

(j) Bookmarks

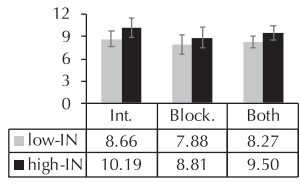

(c) Queries

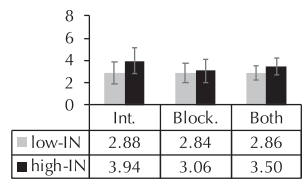

(g) Queries w/o scrolls

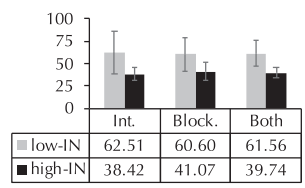

(k) Click-to-bookmark time

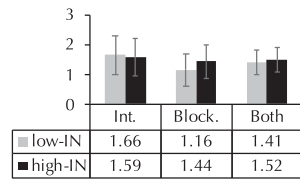

(d) Queries w/o clicks

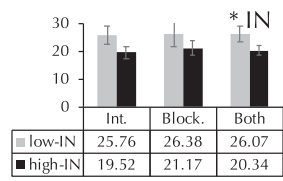

(h) Time b/w events

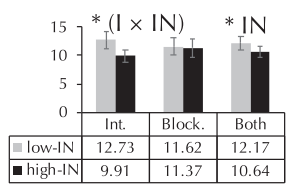

(I) Query-to-click time

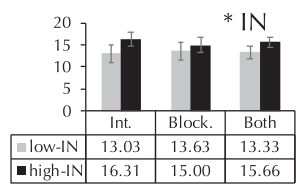

(m) Clicks

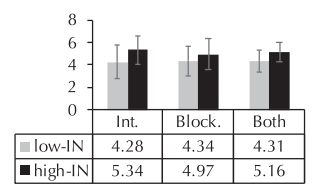

(n) Clicks w/o Bookmarks

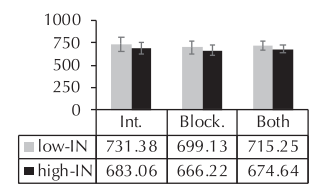

(o) Completion time

Fig. 7. Effects of inhibition group (low-IN, high-IN) and interface (Int. = interleaved, Block. = blocked) on participants' search behaviors. Symbol * IN denotes a significant main effect of inhibition group, and symbol $*(I \times I N)$ denotes a significant interaction effect of interface and inhibition group $(p<0.05)$.

(1) queries with repeated intent $(\beta=1.545$, S.E. $=0.719, p=0.034)$

(2) queries $(\beta=2.030$, S.E. $=1.008, p=0.047)$

(3) quick query reformulations $(\beta=2.526$, S.E. $=0.937, p=0.008)$

(4) queries without mouseovers $(\beta=0.559$, S.E. $=0.275, p=0.045)$

(5) clicks without bookmarks $(\beta=2.487$, S.E. $=0.976, p=0.012)$

The first four measures had the same trend. That is, with the interleaved interface, all measures were higher for low-PS participants than high-PS participants. Conversely, with the blocked interface, the differences between low- and high-PS participants were less pronounced.

For the number of clicks without any bookmarks, we see a different trend. For this measure, the differences between low- and high-PS participants were greater with the blocked interface than with the interleaved interface.

Summary: Overall, our results suggest that low-PS participants had more difficulty identifying relevant results on the SERP. Compared to high-PS participants, low-PS participants issued more queries and had more queries where they did not engage with the search results (e.g., queries without bookmarks or scroll events).

Furthermore, our results suggest that low-PS participants had greater difficulty with the interleaved interface than with the blocked interface. Specifically, with the interleaved interface, low-PS participants issued significantly more queries, had quicker query reformulations, had more queries 
with a repeated intent, and had more queries without any mouseover events. Conversely, with the blocked interface, the differences between low- and high-PS participants were less pronounced.

Interestingly, with the blocked interface, high-PS participants had more clicks (not significant) and more clicks without a bookmark (significant, $p=0.012$ ). Our interpretation of this result is that high-PS participants were more capable of exploiting the fixed layout of the blocked interface to examine more results and were therefore more "selective" when bookmarking pages.

\subsection{Inhibition: Main and Interaction Effects}

Figure 7 shows the means and 95\% confidence intervals for our 15 behavioral measures according interface condition and inhibition group.

Main Effects: Inhibition had a significant main effect for five measures:

(1) quick query reformulations $(\beta=1.778$, S.E. $=0.833, p=0.037)$

(2) time between events $(\beta=-6.332$, S.E. $=2.672, p=0.022)$

(3) bookmarks $(\beta=1.793$, S.E. $=0.735, p=0.018)$

(4) query-to-click time $(\beta=-2.733$, S.E. $=1.172, p=0.024)$

(5) $\operatorname{clicks}(\beta=3.334$, S.E. $=1.466, p=0.027)$

Inhibition also had a strong (but not significant) effect on the time participants took to produce the first bookmark $(\beta=-39.404$, S.E. $=20.095, p=0.055)$. According to all these measures, high-IN participants worked at a faster pace (e.g., took less time between search actions) and performed more actions (e.g., more clicks and bookmarks) and than low-IN participants.

Interaction Effects: Inhibition and interface condition had a significant interaction effect on the avg. query to click time $(\beta=2.436$, S.E. $=1.132, p=0.034)$. With the interleaved interface, high-IN participants took significantly less time to produce the first SERP click after issuing a query. Conversely, with the blocked interface, high- and low-IN participants took similar amounts of time to produce the first SERP click.

Summary: Our interpretation of these results is that high-IN participants worked at a faster pace than low-IN participants because they were more effective at filtering (or inhibiting attention to) task-irrelevant information on SERPs and landing pages. Additionally, we observed a significant interaction effect for one measure: time to first SERP click (if any). This result suggests that low-IN participants were more distracted by non-relevant information with the interleaved interface than with the blocked interface.

\section{DISCUSSION}

In this section, we summarize our results, compare them with results from prior work, and discuss their implications. We also discuss caveats and limitations of our study. Table 7 provides a summary of the main trends observed in our results.

\subsection{Effects of Working Memory}

Summary: Working memory had significant effects on several measures related to workload (RQ1) and user engagement (RQ2). Compared to low-WM participants, high-WM participants reported significantly lower levels of physical demand and frustration (Figure 3), as well as higher levels of perceived usability and reward (Figure 4). Interestingly, working memory and interface also had a significant interaction effect on mental demand. Low-WM participants reported similar levels of mental demand with both interfaces, but high-WM participants reported lower levels of mental demand with the blocked interface. Working memory did not have any significant effects on participants' search behaviors (RQ3). That said, our results suggest that high-WM participants had slightly better outcomes (e.g., more bookmarks, $p=0.060$ ). 
Table 7. Summary of Main and Interaction Effects for Measures Related to Workload (RQ1), Engagement (RQ2), and Search Behaviors (RQ3)

\begin{tabular}{|c|c|c|c|}
\hline & Workload (RQ1) & Engagement (RQ2) & Search Behaviors (RQ3) \\
\hline Interface (I) & - & - & $\begin{array}{l}\text { Participants issued more } \\
\text { queries with the } \\
\text { interleaved interface. }\end{array}$ \\
\hline Working Memory (WM) & $\begin{array}{l}\text { High-WM participants } \\
\text { reported less physical } \\
\text { demand and frustration. }\end{array}$ & $\begin{array}{c}\text { High-WM participants } \\
\text { reported greater } \\
\text { perceived usability and } \\
\text { reward. } \\
\end{array}$ & - \\
\hline Perceptual Speed (PS) & - & - & $\begin{array}{l}\text { Low-PS participants had } \\
\text { more difficulty finding } \\
\text { relevant results on } \\
\text { SERPs (i.e., behavioral } \\
\text { measures related to } \\
\text { Factor 1). }\end{array}$ \\
\hline Inhibition (IN) & - & - & $\begin{array}{c}\text { Low-IN participants } \\
\text { interacted at a slower } \\
\text { pace and accomplished } \\
\text { less (i.e., behavioral } \\
\text { measures related to } \\
\text { Factor 2). }\end{array}$ \\
\hline I x WM & $\begin{array}{l}\text { High-WM participants } \\
\text { reported less mental } \\
\text { demand with the } \\
\text { blocked interface. }\end{array}$ & - & - \\
\hline Ix PS & - & - & $\begin{array}{c}\text { Differences in SERP } \\
\text { engagement between } \\
\text { low- and high-PS } \\
\text { participants were } \\
\text { greater with the } \\
\text { interleaved interface. } \\
\text { Low-IN participants had } \\
\text { more query } \\
\text { abandonment with the } \\
\text { interleaved interface. } \\
\end{array}$ \\
\hline I x IN & - & - & $\begin{array}{l}\text { Differences in pace of } \\
\text { interaction between } \\
\text { low- and high-IN } \\
\text { participants were } \\
\text { greater with the } \\
\text { interleaved interface. } \\
\text { Low-IN participants had } \\
\text { longer query-to-click } \\
\text { times with the } \\
\text { interleaved interface. }\end{array}$ \\
\hline
\end{tabular}

Factors 1 and 2 in the table refer to the factor analysis in Section 3.6.

Interpretations: With respect to working memory, our results suggest three important trends. First, high-WM participants experienced lower levels of workload and higher levels of engagement than low-WM participants. Second, high-WM participants experienced lower levels of mental demand with the blocked interface. One possible explanation is that high-WM participants were more effective at keeping the fixed layout of the blocked interface in active memory and were able 
to use this information to focus their attention on relevant blocks of results. Finally, high-WM participants searched with slightly more intensity than low-WM participants. Possibly for this reason, high-WM participants bookmarked more pages $(p=0.060)$ and reported greater levels of reward $(p=0.006)$ than low-WM participants.

Comparisons with Prior Work: Our results suggest that high-WM participants searched more intensely and that low-WM participants were more likely to engage in satisficing behaviors (e.g., bookmarked fewer pages). This trend is consistent with prior studies by Gwizdka [27, 29], in which high-WM participants exerted more effort in demanding situations. In Gwizdka [27], while completing complex tasks, high-WM participants issued more queries and had longer completion times than low-WM participants. Similarly, in Gwizdka [29], while completing complex tasks, high-WM participants performed more actions and spent more time reading content pages (particularly toward the end of the session) than low-WM participants.

Our results also suggest that high-WM participants were perhaps more effective at leveraging the fixed layout of the blocked interface to focus their attention on relevant blocks of results and therefore experienced less mental demand. Gwizdka [27] had participants interact with two systems: a baseline system and an experimental system that summarized the search results using a tag cloud (with clickable terms that could be used as filters). High-WM participants were able to leverage the experimental system's tag cloud feature to complete tasks faster, but low-WM participants had similar completion times with both interfaces. Combined with our results, one possible explanation is that high-WM users are generally more effective at leveraging interface features and characteristics to improve their search performance.

\subsection{Effects of Perceptual Speed}

Summary: Perceptual speed did not have significant effects on measures related to workload (RQ1) and user engagement (RQ2) but had several significant effects on participants' search behaviors (RQ3). Based on the factor analysis presented in Section 3.6, perceptual speed had the strongest effects on behavioral measures associated with the extent to which participants had difficulty finding relevant results on the SERP (Factor 1). In terms of main effects, low-PS participants issued more queries, had more queries without any scroll events or bookmarks, had more queries with a repeated search intent (same as a previously issued query), and had more quick reformulations.

Additionally, in terms of interaction effects, our results suggest that low-PS participants had greater difficulty finding relevant results with the interleaved interface. With the interleaved interface, low-PS participants issued more queries, had more queries without any mouseover events, had more queries with a repeated intent, and had more quick reformulations. For these four behavioral measures, high-PS participants had comparable values across both interfaces.

Interpretations: With respect to perceptual speed, our results can be interpreted from two perspectives. One perspective is that high-PS participants were equally effective at finding relevant results with both interfaces. Another perspective is that the blocked layout helped low-PS participants find relevant results as effectively as high-PS participants. That said, while perceptual speed impacted participants' search behaviors, it did not impact outcomes related to workload and engagement. This result suggests that cognitive abilities can impact users' behaviors without impacting post-task perceptions.

Comparisons with Prior Work: Our results have similarities and differences with results from prior studies. In our case, high-PS were more effective at finding relevant results on SERPs. This trend is consistent with previous studies $[1,14]$, in which high-PS participants performed better on search activities requiring visually scanning. In Al-Maskari and Sanderson [1], high-PS participants took significantly less time to find the first relevant result. Similarly, in Brennan et al. [14], high-PS participants had significantly more SERP clicks and URLs visited. 
Additionally, in our case, low-PS participants had more difficulty finding relevant results with the interleaved versus blocked interface. Turpin et al. [54] examined the effects of perceptual speed with two different interfaces: an aggregated and non-aggregated interface. High-PS participants had similar completion times with both interfaces, but low-PS participants took significantly longer with the aggregated interface (more "cluttered" than the non-aggregated one).

Finally, in our case, perceptual speed did not have strong effects on post-task perceptions of workload and engagement. This result is inconsistent with prior work [14, 54]. In Brennan et al. [14], low-PS participants reported higher levels workload, and in Turpin et al. [54], low-PS participants reported lower levels of engagement. There are two possible explanations for these differences. First, while our participants were instructed to bookmark pages, participants in both these prior studies were instructed to create an actual response for the task, which likely increased the task difficulty. Second, in Turpin et al. [54], participants completed tasks that were more complex than our comparative tasks. ${ }^{17}$ Thus, it is possible that our tasks were difficult enough for lowPS participants to exhibit different behaviors but not difficult enough to impact their post-task perceptions.

\subsection{Effects of Inhibition}

Summary: Similarly to perceptual speed, inhibition did not have significant effects on measures related to workload (RQ1) and user engagement (RQ2) but had several significant effects on participants' search behaviors (RQ3). Based on the factor analysis presented in Section 3.6, inhibition had the strongest effects on behavioral measures associated with participants' pace of interaction (Factor 2). High-IN participants worked at a faster pace than low-IN participants. In terms of main effects, high-IN participants had more quick reformulations, took less time between events, took less time to bookmark the first page, and took less time to click on a search result after issuing a query. Given their faster pace of interaction, high-IN participants also explored more information (i.e., more SERP clicks) and had better outcomes (i.e., more bookmarks).

Additionally, in terms of interaction effects, our results suggest that low-IN participants were slower with the interleaved versus blocked interface. With the interleaved interface, low-IN participants took longer to produce a click after issuing a query. Conversely, high-IN participants took similar amounts of time with both interfaces.

Interpretations: Our results suggest that high-IN participants were able to work at a faster pace and accomplish more. One possible explanation is that high-IN participants were more effective at ignoring (or inhibiting attention to) non-relevant information on SERPs and landing pages. Our results also suggest that, compared to high-IN participants, low-IN participants were slightly more impacted by the more "cluttered" layout of the interleaved interface. In other words, compared to high-IN participants, low-IN participants were possibly more distracted by non-relevant results with the interleaved interface.

Comparisons with Prior Work: To our knowledge, prior studies have not investigated the effects of inhibitory attention control (or selective attention) on search behaviors or outcomes.

\subsection{Implications for Aggregated Search Systems (and Beyond)}

Aggregated search systems integrate results from different sources in a unified presentation. In this respect, aggregated search systems must decide not only which results to present but also how to present them. In our study, we focused on two interfaces. Compared to the interleaved interface, the blocked interface used the Gestalt principles proximity, similarity, and common region to more

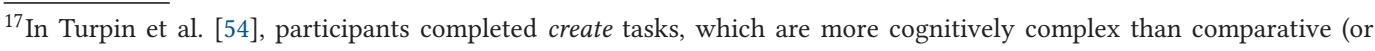
analyze) tasks [35].
} 
clearly distinguish between results from different back-end systems (web, images, news, shopping, and video). Our results have three important implications for aggregated search (and beyond).

Systems Based on Gestalt Principles Provide Benefits: First, our results suggest that aggregated search systems designed based on Gestalt principles (e.g., the blocked interface) can benefit users with specific cognitive abilities (i.e., high-WM, low-PS, and low-IN users). In our study, highWM participants reported lower mental demand with the blocked interface, possibly because they were more effective at keeping fixed layout of the blocked interface in active memory. Based on several behavioral measures, low-PS participants were less effective at finding relevant results on SERPs with the interleaved interface. Possibly, the blocked interface allowed low-PS participants to perform less visual scanning by allowing them to focus their attention on relevant regions of the SERP. Finally, low-IN participants had longer query-to-click times with the interleaved interface. Perhaps, the blocked interface helped low-IN participants to avoid being distracted by results from non-relevant sources.

The above trends resonate with prior work. Arguello and Capra [7] investigated factors that moderate the level of "spillover" between different groups of results in an aggregated SERP. Results found that Gestalt principles (e.g., proximity and common region) can help reduce negative spillover effects (e.g., non-relevant images influencing users to ignore other results). In other words, Arguello and Capra [7] found that Gestalt principles can be used to nudge users to treat results from different verticals as being separate and independent.

Considering Cognitive Abilities when Interpreting Behavioral Data: Search systems use behavioral measures to infer outcomes related to a user's satisfaction and/or performance (e.g., in $\mathrm{A} / \mathrm{B}$ testing or online learning). In our study, participants with different cognitive abilities (perceptual speed and inhibition) exhibited different behaviors. Thus, our results suggest that knowledge of users' cognitive abilities may lead to more accurate inferences from behavioral signals. In our study, low-PS participants had more queries where they did not engage with the search results. In this respect, an abandoned query may be a weaker indicator of poor search results for low-PS versus high-PS searchers. Similarly, in our study, low-IN participants interacted at a slower pace than high-IN participants. In this respect, a longer dwell time on a clicked result may be a weaker indicator of relevance (i.e., a "satisfied" click) for low-IN versus high-IN searchers.

Inferring Cognitive Abilities: Above, we argue that systems should consider users' cognitive abilities in deciding how to display results and interpreting behavioral measures. Of course, both of these goals would require inferring users' cognitive abilities from their interactions with a system. Our results suggest that some cognitive abilities may be easier to infer than others. In our case, compared to working memory, perceptual speed and inhibition had stronger effects on search behaviors, suggesting that they might be easier to infer. This is perhaps because visual scanning and attention control are cognitive activities that users typically perform on SERPs. Working memory is a higher-level ability that may impact higher-level activities such as planning, regulating, inferring relations between concepts, and recognizing/addressing knowledge gaps. Additional research is needed to further understand the impact of working memory on specific search-related activities and their corresponding behaviors.

Prior work also suggests that task complexity plays an important role on the extent to which working memory impacts behaviors [27, 29]. Specifically, these studies have found that high-WM users exert more effort than low-WM participants during complex tasks. This points to yet another potential challenge in inferring working memory ability-it may require also inferring the inherent complexity of a user's current task.

\subsection{Caveats and Limitations}

Our study has several limitations worth noting. 
Task characteristics: First, in this study, we were not interested in investigating task effects. Thus, participants completed tasks that were fairly consistent-comparative tasks that required bookmarking at least 10 relevant pages. Our tasks had two important characteristics. First, they were not highly complex tasks. ${ }^{18}$ Second, our tasks required a fair amount of visual scanning on SERPs. Further work is needed to understand how our task characteristics may have influenced our results. Working memory may have greater effects on behavioral measures for more complex tasks. Similarly, perceptual speed and inhibition may reduced effects on search behaviors for tasks requiring less visual scanning at the SERP level.

Interfaces: A second limitation of our study stems from the two interfaces investigated. First, the interleaved and blocked interfaces were very different from each other. In fact, one might view these two interfaces as being at extreme ends of a continuum. Additional research is needed to understand how individual Gestalt principles (similarity, proximity, and common region) may have an effect on search behaviors and outcomes.

Finally, neither interface positioned results from specific verticals based on their relevance to the input query. The blocked interface always displayed vertical results in the same positions on the SERP and the interleaved interface inserted vertical results (consistent with their original ranking) between web results in a pseudo-random fashion. Our positioning strategies were motivated by two criteria. First, we wanted the blocked interface to have a fixed (i.e., query-independent) layout. Second, we wanted both interfaces to respond quickly to participants' queries. That said, neither of these positioning strategies mirror how current web search portals (e.g., Google) display verticalsby predicting the user's vertical intent [4]. Future work is needed to understand the impact of Gestalt principles when vertical results are made more/less visually salient (e.g., ranked higher on the SERP) based on the predicted vertical intent of a user.

\section{CONCLUSION}

In this article, we reported on a study that investigated the effects of two aggregated search interfaces (blocked vs. interleaved) and three cognitive abilities (working memory, perceptual speed, and inhibition) on three types of outcomes: (RQ1) participants' levels of workload, (RQ2) user engagement, and (RQ3) search behaviors. Our two interfaces displayed the same results but varied in the extent to which the visual layout distinguished between results from different sources (web, images, news, shopping, and video). Specifically, the blocked interface displayed results from the same source as a group, displayed groups in the same positions for every query, and displayed each group encircled in a border.

Our results found several important trends. Perceptual speed (PS) and inhibition (IN) did not have significant effects on the levels of workload and engagement reported by participants but had significant effects on their search behaviors. In terms of perceptual speed, low-PS participants issued more queries where they did not engage with the search results, suggesting that they had more difficulty scanning for and identifying relevant results on the SERP. In terms of inhibition, low-IN participants searched at a slower pace, suggesting that they were more easily distracted by non-relevant information. Importantly, both trends were more pronounced with the interleaved interface, which was more visually "cluttered" than the blocked interface.

Working memory (WM) had significant effects on several measures related to workload and engagement. Regardless of the interface, high-WM participants reported significantly lower levels of physical demand and frustration, as well as higher levels of perceived usability and

\footnotetext{
${ }^{18}$ Within the cognitive complexity framework [35], comparative tasks fall under analyze tasks, which have an intermediate level of complexity. More complex tasks include tasks that require comparing alternatives and making a decision (evaluate tasks), as well as tasks that require creating a new solution to a problem (create tasks).
} 
reward. Furthermore, with the blocked interface, high-WM participants reported significantly lower levels of mental demand (i.e., an interaction effect). One explanation is that high-WM participants were better able to keep the fixed layout of the blocked interface in active memory and used this information to focus their attention on relevant blocks of results throughout the search session.

Working memory did not have strong effects on participants' search behaviors. As one exception, high-WM participants were able to produce slightly more bookmarks than low-WM participants (not significant, $p=0.060$ ). This trend resonates with prior studies [27, 29], which found that high-WM searchers tend to exert more effort than low-WM searchers in demanding situations.

Our results have important implications for IR research. First, our results suggest that aggregated interfaces based on Gestalt principles (i.e., the blocked vs. interleaved interface) may provide benefits for users with specific abilities-users who are slower at visual scanning, users who are more easily distracted by non-relevant information, and users who are better able to keep a fixed layout in active memory. Second, our results suggest that knowledge of users' specific abilities may lead to better inferences about search outcomes (e.g., satisfaction) from behavioral data (e.g., in A/B tests). For example, based on our results, abandoned queries may be a weaker signal of poor search results for low-PS versus high-PS users. Finally, our results suggest that some cognitive abilities may be easier to infer than others based on behavioral data. In our case, perceptual speed and inhibition had stronger effects on participants' behaviors than working memory. One possibility is that visual scanning and attention control are low-level cognitive activities that users must necessarily perform on SERPs. Further research is needed to understand how working memory (a higher-level ability) influences specific search-related activities and related behaviors.

In terms of future work, many open questions remain. In the near term, in this article, we did not study task effects. Participants completed tasks that were not highly complex (i.e., comparative tasks) and required simply bookmarking pages (versus making a final decision or proposing a solution to a problem). For more complex tasks, working memory may have stronger effects on search behaviors, and perceptual speed and inhibition may have stronger effects on perceptions of workload and engagement. Furthermore, our two aggregated search layouts were very different from each other. Additional research is needed to understand how more subtle differences in the interface may influence users with different abilities.

Looking farther into the future, there are deeper questions to consider. Search is a cognitive activity involving visual scanning, attention control, memorization, and making higher-level inferences. Therefore, it seems worthwhile to further understand how different search-related activities rely on specific cognitive abilities. Furthermore, future research should consider how specific task characteristics and contextual factors (e.g., multitasking) may also play a role. Progress in this area may inform the design and implementation of personalized displays, interactions, and scaffolding tools (e.g., note-taking tools) to support searchers based on their unique abilities.

\section{APPENDIX}

\section{A SUMMARY OF STATISTICAL MODELS}

Tables 8-10 summarize the output of each mixed-effects model for all outcome measures pertaining to RQ1-RQ3, respectively. Tables 8-10 also include the marginal and conditional $R^{2}$ values, computed as described in Nakagawa and Schielzeth [44]. The marginal $R^{2}$ values indicate the percentage of the variance explained by all fixed factors (without random intercepts based on the participant and task) and the conditional $R^{2}$ values indicate the percentage of the variance explained by the whole model, including all fixed factors and random factors. 
Table 8. Summary of Multilevel Models for RQ1 (Workload)

\begin{tabular}{lccccccc}
\hline & Mental D. & Physical D. & Temporal D. & Effort & Failure & Frustration & Overall Workload \\
\hline Intercept & $3.144^{*}$ & $2.119^{*}$ & $4.012^{*}$ & $3.604^{*}$ & $3.381^{*}$ & $3.022^{*}$ & $3.214^{*}$ \\
Interface & 0.263 & 0.358 & -0.177 & 0.115 & -0.071 & -0.197 & 0.048 \\
PS group & -0.138 & 0.111 & -0.433 & 0.017 & -0.188 & 0.035 & -0.098 \\
WM group & -0.130 & $-0.757^{*}$ & -0.409 & -0.253 & -0.570 & $-1.239^{*}$ & -0.559 \\
IN group & 0.035 & -0.165 & -0.515 & -0.360 & -0.675 & -0.255 & -0.324 \\
Interface $\times$ PS group & 0.295 & -0.415 & -0.011 & -0.167 & 0.360 & 0.296 & 0.058 \\
Interface $\times$ WM group & $-0.949^{*}$ & -0.058 & -0.545 & -0.581 & -0.419 & -0.020 & -0.431 \\
Interface $\times$ IN group & -0.583 & -0.248 & 0.172 & 0.113 & 0.251 & -0.092 & -0.061 \\
\hline AIC & 461.60 & 388.70 & 476.00 & 489.10 & 467.30 & 466.70 & 363.20 \\
BIC & 492.90 & 420.00 & 507.40 & 520.50 & 498.70 & 498.10 & 394.50 \\
$R^{2}$ (marginal) & 0.085 & 0.117 & 0.093 & 0.047 & 0.100 & 0.166 & 0.148 \\
$R^{2}$ (conditional) & 0.401 & 0.567 & 0.487 & 0.276 & 0.366 & 0.335 & 0.555 \\
\hline
\end{tabular}

Symbol “*” denotes statistically significant $\beta$ and intercept values $(p<0.05)$.

Table 9. Summary of Multilevel Models for RQ2 (User Engagement)

\begin{tabular}{lccccc}
\hline & Perceived Usability & Aesthetic Appeal & Focused Attention & Reward & Overall Engagement \\
\hline Intercept & $5.308^{*}$ & $3.051^{*}$ & $4.429^{*}$ & $4.124^{*}$ & $4.229^{*}$ \\
Interface & 0.062 & 0.161 & -0.160 & 0.378 & 0.108 \\
PS group & 0.005 & 0.461 & -0.240 & -0.248 & -0.006 \\
WM group & $0.736^{*}$ & -0.441 & 0.233 & $1.013^{*}$ & 0.385 \\
IN group & -0.075 & 0.067 & -0.046 & 0.484 & 0.109 \\
Interface $\times$ PS group & -0.033 & 0.032 & 0.356 & -0.161 & 0.050 \\
Interface $\times$ WM group & 0.407 & 0.239 & -0.351 & -0.371 & -0.019 \\
Interface $\times$ IN group & 0.079 & -0.313 & 0.196 & -0.030 & -0.020 \\
\hline AIC & 388.70 & 409.80 & 405.20 & 418.70 & 302.90 \\
BIC & 420.10 & 441.20 & 436.50 & 450.00 & 334.30 \\
$R^{2}$ (marginal) & 0.174 & 0.041 & 0.009 & 0.119 & 0.048 \\
$R^{2}$ (conditional) & 0.436 & 0.685 & 0.683 & 0.558 & 0.612 \\
\hline
\end{tabular}

Symbol “*” denotes statistically significant $\beta$ and intercept values $(p<0.05)$.

Table 10. Summary of Multilevel Models for RQ3 (Search Behaviors)

\begin{tabular}{lcccc}
\hline & Queries w/o Books. & Repeated Intent & Queries & Queries w/o Clicks \\
\hline Intercept & $4.469^{*}$ & $2.709^{*}$ & $9.408^{*}$ & $1.971^{*}$ \\
Interface & -1.470 & -1.120 & $-2.020^{*}$ & -0.595 \\
PS group & $-1.741^{*}$ & $-1.430^{*}$ & $-2.401^{*}$ & -0.820 \\
WM group & 0.464 & 0.657 & 1.013 & 0.210 \\
IN group & 0.778 & 0.964 & 1.468 & -0.120 \\
Interace x PS group & 1.225 & $1.545^{*}$ & $2.030^{*}$ & 0.249 \\
Interace x WM group & 0.296 & 0.079 & 0.537 & -0.050 \\
\hline & & & (Continued)
\end{tabular}


Table 10. Continued

\begin{tabular}{lcccc}
\hline & Queries w/o Books. & Repeated Intent & Queries & Queries w/o Clicks \\
\hline Interface x IN group & -0.088 & -0.143 & -0.479 & 0.394 \\
\hline AIC & 631.00 & 593.70 & 680.80 & 493.30 \\
BIC & 662.30 & 625.10 & 712.20 & 524.70 \\
$R^{2}$ (marginal) & 0.093 & 0.098 & 0.139 & 0.059 \\
$R^{2}$ (conditional) & 0.402 & 0.349 & 0.380 & 0.333 \\
\hline & Quick Reform. & Queries w/o Mouse. & Queries w/o Scrolls & Time b/w Events \\
\hline Intercept & $4.178^{*}$ & $0.709^{*}$ & $3.995^{*}$ & $26.681^{*}$ \\
Interface & -1.445 & -0.435 & -0.942 & 3.462 \\
PS group & $-2.746^{*}$ & -0.399 & $-2.575^{*}$ & 1.148 \\
WM group & 1.444 & 0.050 & 0.617 & -3.547 \\
IN group & $1.778^{*}$ & 0.072 & 0.811 & $-6.332^{*}$ \\
Interace x PS group & $2.526^{*}$ & $0.559^{*}$ & 1.317 & -3.197 \\
Interace x WM group & -0.083 & -0.003 & 0.110 & -2.574 \\
Interface x IN group & -0.815 & -0.121 & -0.496 & 0.542 \\
\hline AIC & 659.90 & 323.00 & 613.30 & 921.40 \\
BIC & 691.30 & 354.40 & 644.70 & 952.80 \\
$R^{2}$ (marginal) & 0.176 & 0.059 & 0.155 & 0.162 \\
$R^{2}$ (conditional) & 0.389 & 0.096 & 0.413 & 0.525 \\
\hline & $B 00 k$. & -10 &
\end{tabular}

\begin{tabular}{|c|c|c|c|c|}
\hline & First Book. Time & Bookmarks & Click-to-Book Time & Query-to-Click Time \\
\hline Intercept & $116.629^{*}$ & $7.606^{*}$ & $59.805^{*}$ & $12.231^{*}$ \\
\hline Interface & -4.998 & 0.407 & 5.378 & -0.535 \\
\hline PS group & -4.364 & 1.328 & 3.797 & 1.436 \\
\hline WM group & -11.093 & 1.438 & 1.848 & -0.563 \\
\hline IN group & -39.404 & $1.793^{*}$ & -23.738 & $-2.733^{*}$ \\
\hline Interace $\mathrm{x}$ PS group & -10.229 & -1.360 & -5.578 & -0.608 \\
\hline Interace $\mathrm{x}$ WM group & -13.100 & 1.298 & -10.278 & -0.500 \\
\hline Interface x IN group & 17.729 & -0.650 & 3.560 & $2.436^{*}$ \\
\hline$\overline{\mathrm{AIC}}$ & 1467.80 & 615.10 & 1333.70 & 720.70 \\
\hline $\mathrm{BIC}$ & 1499.20 & 646.50 & 1365.10 & 752.00 \\
\hline$R^{2}$ (marginal) & 0.067 & 0.205 & 0.062 & 0.084 \\
\hline \multirow[t]{2}{*}{$R^{2}$ (conditional) } & 0.307 & 0.523 & 0.443 & 0.426 \\
\hline & Clicks & Clicks w/o Books & Completion Time & \\
\hline$\overline{\text { Intercept }}$ & $12.609^{*}$ & $4.686^{*}$ & $729.040^{*}$ & \\
\hline Interface & -0.524 & -0.733 & -27.220 & \\
\hline PS group & 0.824 & -0.390 & 45.960 & \\
\hline WM group & 0.024 & -0.545 & -50.110 & \\
\hline IN group & $3.334^{*}$ & 0.978 & -47.920 & \\
\hline Interace $\mathrm{x}$ PS group & 1.254 & $2.487^{*}$ & -37.970 & \\
\hline Interace $\mathrm{x}$ WM group & 1.121 & -0.893 & 37.730 & \\
\hline Interface $\mathrm{x}$ IN group & -1.758 & -0.286 & 14.080 & \\
\hline$\overline{\mathrm{AIC}}$ & 768.30 & 688.50 & 1661.50 & \\
\hline $\mathrm{BIC}$ & 799.60 & 719.90 & 1692.90 & \\
\hline$R^{2}$ (marginal) & 0.093 & 0.068 & 0.031 & \\
\hline$R^{2}$ (conditional) & 0.464 & 0.477 & 0.648 & \\
\hline
\end{tabular}

Symbol “*” denotes statistically significant $\beta$ and intercept values $(p<0.05)$. 


\section{REFERENCES}

[1] Azzah Al-Maskari and Mark Sanderson. 2011. The effect of user characteristics on search effectiveness in information retrieval. Inf. Process. Manage. 47, 5 (2011), 719-729.

[2] Bryce Allen. 1992. Cognitive differences in end user searching of a CD-ROM index. In SIGIR. ACM, 298-309.

[3] Bryce Allen. 1994. Perceptual speed, learning and information retrieval performance. In SIGIR. Springer-Verlag, 7180 .

[4] Jaime Arguello. 2017. Aggregated search. Found. Trends Inf. Retriev. 10, 5 (2017), 365-502.

[5] Jaime Arguello and Robert Capra. 2012. The effect of aggregated search coherence on search behavior. In CIKM. ACM, 1293-1302.

[6] Jaime Arguello and Robert Capra. 2014. The effects of vertical rank and border on aggregated search coherence and search behavior. In CIKM. ACM, 539-548.

[7] Jaime Arguello and Rob Capra. 2016. The effects of aggregated search coherence on search behavior. Trans. Inf. Syst. 35,1 (2016), 2:1-2:30.

[8] Jaime Arguello, Robert Capra, and Wan-Ching Wu. 2013. Factors affecting aggregated search coherence and search behavior. In CIKM. ACM, 1989-1998.

[9] Jaime Arguello, Fernando Diaz, Jamie Callan, and Jean-Francois Crespo. 2009. Sources of evidence for vertical selection. In SIGIR. ACM, 315-322.

[10] International Dyslexia Association. 2017. Dyslexia Basics. Retrieved August 8, 2018 from https://dyslexiaida.org/ dyslexia-basics/.

[11] R. H. Baayen, D. J. Davidson, and D. M. Bates. 2008. Mixed-effects modeling with crossed random effects for subjects and items. F. Mem. Lang. 59, 4 (2008), 390-412.

[12] Kara L. Bopp and Paul Verhaeghen. 2005. Aging and verbal memory span: A meta-analysis. F. Gerontol. B 605 (2005), P223-33.

[13] Horatiu Bota, Ke Zhou, and Joemon M. Jose. 2016. Playing your cards right: The effect of entity cards on search behaviour and workload. In CHIIR. ACM, 131-140.

[14] Kathy Brennan, Diane Kelly, and Jaime Arguello. 2014. The effect of cognitive abilities on information search for tasks of varying levels of complexity. In IIiX. ACM, 165-174.

[15] Rob Capra, Jaime Arguello, Heather O’Brien, Yuan Li, and Bogeum Choi. 2018. The effects of manipulating task determinability on search behaviors and outcomes. In SIGIR. ACM, 445-454.

[16] Rob Capra, Jaime Arguello, and Yinglong Zhang. 2017. The effects of search task determinability on search behavior. In ECIR. Springer, 108-121.

[17] Danqi Chen, Weizhu Chen, Haixun Wang, Zheng Chen, and Qiang Yang. 2012. Beyond ten blue links: Enabling user click modeling in federated web search. In WSDM. ACM, 463-472.

[18] Lisa Cothran and Randy Larsen. 2008. Comparison of inhibition in two timed reaction tasks: The color and emotion stroop tasks. F. Psychol. 142, 4 (2008), 159-174.

[19] Adele Diamond. 2013. Executive functions. Annu. Rev. Psychol. 64 (2013), 135-168.

[20] Fernando Diaz, Ryen White, Georg Buscher, and Dan Liebling. 2013. Robust models of mouse movement on dynamic web search results pages. In CIKM. ACM, 1451-1460.

[21] Susan Dumais, Edward Cutrell, and Hao Chen. 2001. Optimizing search by showing results in context. In CHI. ACM, 277-284.

[22] Ruth B. Ekstrom, John W. French, Harry H. Harman, and Diran Dermen. 1976. Manual for Kit of Factor-Referenced Cognitive Tests. ETS.

[23] John E. Fisk and Peter Warr. 1996. Age and working memory: The role of perceptual speed, the central executive, and the phonological loop. Psychol. Aging 11, 2 (1996).

[24] Adam Fourney, Meredith Ringel Morris, Abdullah Ali, and Laura Vonessen. 2018. Assessing the readability of web search results for searchers with dyslexia. In SIGIR. ACM, 1069-1072.

[25] Greg Francis, Ian Neath, and Danien VanHorn. 2007. Coglab on a CD, Version 2.0. Cengage Learning.

[26] Susan M. Gass, Jennifer N. Behney, and Baburhan Uzum. 2013. Inhibitory Control, Working Memory and L2 Interaction. Springer, Berlin, 91-114.

[27] Jacek Gwizdka. 2009. What a difference a tag cloud makes: Effects of tasks and cognitive abilities on search results interface use. Inf. Res. 14, 4 (2009).

[28] Jacek Gwizdka. 2010. Using stroop task to assess cognitive load. In ECCE. ACM, 219-222.

[29] Jacek Gwizdka. 2017. I can and so i search more: Effects of memory span on search behavior. In CHIIR. ACM, 341-344.

[30] Sandra G. Hart and Lowell E. Staveland. 1988. Development of NASA-TLX (task load index): Results of empirical and theoretical research. In Human Mental Workload. Advances in Psychology, Vol. 52. North-Holland, 139-183.

[31] Peter J. Houx, Jellemer Jolles, and Fred W. Vreeling. 1993. Stroop interference: Aging effects assessed with the stroop color-word test. Exp. Aging Res. 19, 3 (1993), 209-224. 
[32] J. Everatt, J. Warner, T. R. Miles, and M. E. Thomson1997. The incidence of stroop interference in dyslexia. Dyslexia 3, 4 (1997), 222-228.

[33] Holmes Joni, Gathercole Susan E., Place Maurice, Dunning Darren L., Hilton Kerry A., and Elliott Julian G.2009. Working memory deficits can be overcome: Impacts of training and medication on working memory in children with ADHD. Appl. Cogn. Psychol. 24, 6 (2009), 827-836.

[34] Michael Kane and Randall Engle. 2003. Working-memory capacity and the control of attention: The contributions of goal neglect, response competition, and task set to stroop interference. f. Exp. Psychol. 132, 1 (2003), 47-70.

[35] Diane Kelly, Jaime Arguello, Ashlee Edwards, and Wan-ching Wu. 2015. Development and evaluation of search tasks for IIR experiments using a cognitive complexity framework. In ICTIR. ACM, 101-110.

[36] Jinyoung Kim and W. Bruce Croft. 2010. Ranking using multiple document types in desktop search. In SIGIR. ACM, $50-57$.

[37] Kyung-Sun Kim and Bryce Allen. 2001. Cognitive and task influences on Web searching behavior. f. Assoc. Inf. Sci. Technol. 53, 2 (2001), 109-119.

[38] K. Koffka. 1935. Principles of Gestalt Psychology. Harcourt, New York.

[39] J. R. Landis and G. G. Koch. 1977. The measurement of observer agreement for categorical data. Biometrics 33, 1 (1977), 159-174.

[40] A. MacFarlane, A. Albrair, C. R. Marshall, and G. Buchanan. 2012. Phonological working memory impacts on information searching: An investigation of dyslexia. In IIiX. ACM, 27-34.

[41] Colin M. MacLeod. 1991. Half a century of research on the stroop effect: An integrative review. Psychol. Bull. 109, 2 (1991), 163-203.

[42] Ilya Markov, Eugene Kharitonov, Vadim Nikulin, Pavel Serdyukov, Maarten de Rijke, and Fabio Crestani. 2014. Vertical-aware click model-based effectiveness metrics. In CIKM. ACM, 1867-1870.

[43] Meredith Ringel Morris, Adam Fourney, Abdullah Ali, and Laura Vonessen. 2018. Understanding the needs of searchers with dyslexia. In CHI. ACM, 35:1-35:12.

[44] Shinichi Nakagawa and Holger Schielzeth. 2012. A general and simple method for obtaining R2 from generalized linear mixed-effects models. Methods Ecol. Evol. 4, 2 (2012), 133-142.

[45] Else Nygren. 1996. Between the clicks: Skilled users scanning of pages. Designing for the Web: Empirical Studies. Redmond, WA, USA.

[46] Heather L. O’Brien, Paul Cairns, and Mark Hall. 2018. A practical approach to measuring user engagement with the refined user engagement scale (UES) and new UES short form. International fournal of Human-Computer Studies (2018).

[47] Center of Decease Control and Prevention. 2015. QuickStats: Percentage of Children and Adolescents Aged 5 to 17 Years with Diagnosed Attention-Deficit/Hyperactivity Disorder (ADHD). Retrieved August 8, 2018 from https:// www.cdc.gov/mmwr/preview/mmwrhtml/mm6433a11.htm.

[48] Stephen E. Palmer. 1992. Common region: A new principle of perceptual grouping. Cogn. Psychol. 24, 3 (1992), 436447.

[49] K. Pawlik. 1966. Concepts and calculations in human cognitive abilities. Handbook of Multivariate Experimental Psychology. Rand McNally.

[50] Ashok Kumar Ponnuswami, Kumaresh Pattabiraman, Qiang Wu, Ran Gilad-Bachrach, and Tapas Kanungo. 2011. On composition of a federated web search result page: Using online users to provide pairwise preference for heterogeneous verticals. In WSDM. ACM, 715-724.

[51] John Stroop. 1935. Studies of interference in serial verbal reactions. f. Exp. Psychol. 18, 6 (1935), 643-662.

[52] Shanu Sushmita, Hideo Joho, Mounia Lalmas, and Robert Villa. 2010. Factors affecting click-through behavior in aggregated search interfaces. In CIKM. ACM, 519-528.

[53] S. Sushmita, B. Piwowarski, and M. Lalmas. 2010. Dynamics of genre and domain intents. In AAIRS. Springer-Verlag, 399-409.

[54] Lauren Turpin, Diane Kelly, and Jaime Arguello. 2016. To blend or not to blend?: Perceptual speed, visual memory and aggregated search. In SIGIR. ACM, 1021-1024.

[55] Chao Wang, Yiqun Liu, Min Zhang, Shaoping Ma, Meihong Zheng, Jing Qian, and Kuo Zhang. 2013. Incorporating vertical results into search click models. In SIGIR. ACM, 503-512.

[56] Wan-Ching Wu, Diane Kelly, and Avneesh Sud. 2014. Using information scent and need for cognition to understand online search behavior. In SIGIR. ACM, 557-566.

Received August 2018; revised March 2019; accepted March 2019 